\title{
CONFORMAL SCALAR PROPAGATION INSIDE THE SCHWARZSCHILD BLACK HOLE
}

\author{
GEORGE TSOUPROS \\ PRIVATE ADDRESS - BEIJING \\ PEOPLE'S REPUBLIC OF CHINA
}

\begin{abstract}
The analytic expression obtained in the preceding project for the massless conformal scalar propagator in the Hartle-Hawking vacuum state for small values of the Schwarzschild radial coordinate above $r=2 M$ is analytically extended into the interior of the Schwarzschild black hole. The result of the analytical extension coincides with the exact propagator for a small range of values of the Schwarzschild radial coordinate below $r=2 M$ and is an analytic expression which manifestly features its dependence on the background space-time geometry. This feature as well as the absence of any assumptions and prerequisites in the derivation render this Hartle-Hawking scalar propagator in the interior of the Schwarzschild black-hole geometry distinct from previous results. The two propagators obtained in the interior and in the exterior region of the Schwarzschild black hole are matched across the event horizon. The result of that match is a massless conformal scalar propagator in the Hartle-Hawking vacuum state which is shown to describe particle production by the Schwarzschild black hole.
\end{abstract}

\section{Introduction}

"The future is not what it used to be!" From Alan Parker's film "Angel Heart"

The intimate relation between the Feynman propagator and the expectation value $<T_{\mu \nu}>$ of the stress tensor in any specific vacuum state is the primary reason that the former is an essential mathematical construct for the study of vacuum activity on curved manifolds. In a black-hole space-time no less important a reason is the relation between higher-order radiative effects and the radiation emitted by black holes in certain vacuum states. Following the construction of a general expression for the Feynman Green function in the exterior region of the Schwarzschild black hole [1] Candelas and Jensen extended that result with Hartle-Hawking boundary conditions in the interior region of that space-time [2]. As is the case in [1] the exact dependence of the Green function on the Schwarzschild radial variable in [2] remains unknown. Yet another approach to the Feynman Green function in the interior of the Schwarzschild black hole has been proposed in [3]. That Green function is essentially coordinate dependent. Its construction exploits $\mathrm{CPT}$ invariance in order to speculate on a possible vacuum state in the interior which conforms with the Boulware vacuum state.

In [4] an analytic expression for the conformal scalar propagator in the Hartle-Hawking vacuum state was provided. That expression features an explicit dependence on the

present e-mail address: landaughost@hotmail.com. 
Schwarzschild black-hole space-time and is a valid approximation to the unknown exact propagator in the exterior region of that space-time for small values of the radial coordinate above $r=2 M$. This project has two objectives. The first is to extend that analytic expression for the Feynman Green function in the interior of the Schwarzschild black hole. Following that extension the second objective is to match the scalar propagators in the interior region and in the exterior region across the event horizon of the Schwarzschild black hole and to establish that the scalar propagator which emerges as a result of that match necessarily describes particle production by the Schwarzschild black hole, in conformity with Hawking radiation. The analytic expression in which the ensuing analysis will eventuate will, likewise, be shown to be a valid approximation to the exact propagator in the interior of the Schwarzschild black hole for a certain range of radii $r<2 M$. As was also the case in [4] an essential advantage of the propagator in which the analysis herein will eventuate is that it renders the short-distance behaviour and the singularity structure of the propagator manifest. In effect, that Green function is especially suited for an analytic evaluation of $\left\langle T_{\mu \nu}\right\rangle$ and of $\left\langle\phi^{2}(x)\right\rangle$ in the interior region of the Schwarzschild black hole as well as for a higher-order perturbative evaluation of the dynamical behaviour of the conformal scalar field in the presence of interactions in the Schwarzschild black-hole space-time.

\section{The Hartle-Hawking Green function}

The Schwarzschild metric is

$$
d s^{2}=-\left(1-\frac{2 M}{r}\right) d t^{2}+\left(1-\frac{2 M}{r}\right)^{-1} d r^{2}+r^{2}\left(d \theta^{2}+\sin ^{2} \theta d \phi^{2}\right)
$$

The analytical extension $\tau=+i t$ of the real-time coordinate $t$ in imaginary values results in a Euclidean, positive definite metric for $r>2 M$. The apparent singularity which persists at $r=2 M$ can be removed by introducing the new radial coordinate [5]

$$
\rho=4 M\left(1-\frac{2 M}{r}\right)^{\frac{1}{2}}
$$

Upon replacing

$$
\beta=4 M
$$

the metric in the new coordinates is

$$
d s^{2}=\rho^{2}\left(\frac{1}{\beta^{2}}\right) d \tau^{2}+\left(\frac{4 r^{2}}{\beta^{2}}\right)^{2} d \rho^{2}+r^{2}\left(d \theta^{2}+\sin ^{2} \theta d \phi^{2}\right)
$$

The coordinate singularity at $r=2 M$ corresponds to the origin $\rho=0$ of polar coordinates and is removed by identifying $\frac{\tau}{4 M}$ with an angular coordinate of period $2 \pi$. In addition, although the curvature singularity at $r=0$ can not be removed by coordinate transformations this procedure can be seen to avoid it altogether along with the entire $r<2 M$ region of the Schwarzschild geometry in real time. This procedure results, therefore, in a complete singularity-free positive definite, Euclidean metric which is periodic 
in the imaginary-time coordinate $\tau$. The period $8 \pi M$ of the imaginary-time coordinate $\tau$ is the underlying cause of the thermal radiation at temperature $T=(8 \pi M)^{-1}$ emitted by the Schwarzschild black hole.

In resulting in a singularity-free positive definite, Euclidean metric for $r \geq 2 M$ this procedure also singles out in that region the Hartle-Hawking Euclidean Green function for the massless scalar field as that solution to 1

$$
\left[-\square_{x_{2}}+\xi R\left(x_{2}\right)\right] D\left(x_{1}, x_{2}\right)=\delta\left(x_{1}, x_{2}\right)
$$

which is uniquely specified by the requirement that (i) $D\left(x_{2}-x_{1}\right)$ be regular on the Euclidean section of (1) - and, thereby, periodic in the imaginary-time coordinate $\tau$ and (ii) vanish as $r \rightarrow \infty$ - and, consequently, as $\rho \rightarrow \beta$. By imposing the requirement $\rho^{2}<<\beta^{2}$ the solution to (5) was, in [4], determined to be

$$
\begin{gathered}
D\left(x_{2}-x_{1}\right)= \\
\frac{2}{\beta} \frac{1}{\sqrt{\rho_{1} \rho_{2}}} \sum_{l=0}^{\infty} \sum_{m=-l}^{l} Y_{l m}\left(\theta_{2}, \phi_{2}\right) Y_{l m}^{*}\left(\theta_{1}, \phi_{1}\right) \sum_{p=0}^{\infty} e^{i \frac{p}{\beta}\left(\tau_{2}-\tau_{1}\right)} \int_{u_{0}[p]}^{\infty} d u \frac{\cos \left[\frac{\pi}{4 \beta}(4 u+2 p+3)\left(\rho_{2}-\rho_{1}\right)\right]}{\pi^{2} u^{2}+4\left(l^{2}+l+1\right)} \\
-\frac{2}{\beta^{\frac{3}{2}}} \frac{1}{\sqrt{\rho_{1}}} \times \\
\sum_{l=0}^{\infty} \sum_{m=-l}^{l} \sum_{p=0}^{\infty} \int_{u_{0}^{\prime}[p]}^{\infty} d u \frac{\cos \left[\frac{\pi}{4 \beta}(4 u+2 p+3)\left(\beta-\rho_{1}\right)\right]}{\pi^{2} u^{2}+4\left(l^{2}+l+1\right)} \frac{J_{p}\left(\frac{2 i}{\beta} \sqrt{l^{2}+l+1} \rho_{2}\right)}{J_{p}\left(2 i \sqrt{l^{2}+l+1}\right)} e^{i \frac{p}{\beta}\left(\tau_{2}-\tau_{1}\right)} Y_{l m}\left(\theta_{2}, \phi_{2}\right) Y_{l m}^{*}\left(\theta_{1}, \phi_{1}\right) ; \\
u_{0}>>p ; u_{0}^{\prime}>>p ; \frac{\pi u}{\beta} \rho_{2,1}>>p
\end{gathered}
$$

with the range of validity for the Green function in (6) specified by

$$
0 \leq \rho_{i}^{2} \leq \frac{\beta^{2}}{100} ; i=1,2
$$

or, equivalently, by 2

$$
2 M \leq r \leq 2.050 M
$$

It is now desirable to, analytically, extend the Euclidean Green function in (6) to the interior region $r<2 M$ of (1). In this region it is not sufficient to pass to imaginary time as that would entail a metric with signature $(-,-,+,+)[2]$. It is therefore necessary to implement the additional change $\theta \rightarrow i \tilde{\theta}$ as a result of which (1) becomes

\footnotetext{
${ }^{1}$ where for the conformally invariant theory it is $\xi=\frac{1}{6}$

2 in 4 the upper bound was inadvertently stated to be $2.0050 \mathrm{M}$ instead of $2.050 \mathrm{M}$. The correct upper bound $2.050 \mathrm{M}$ increases the already large range of validity in (6) by yet one more order of magnitude.
} 


$$
d s^{2}=-\left[\left[\frac{2 M}{r}-1\right] d \tau^{2}+\frac{1}{\frac{2 M}{r}-1} d r^{2}+r^{2}\left(d \tilde{\theta}^{2}+\sinh ^{2} \tilde{\theta} d \phi^{2}\right)\right]
$$

This metric can be seen to have a negative-definite signature for $r<2 M$. The reasons that the Euclidean Green function subjected to the Hartle-Hawking requirement of periodicity in imaginary time can, in the interior region, only be obtained through an analytical extension of the corresponding Green function in the exterior region of the black-hole geometry - as opposed to, through solving (5) for $0<r \leq 2 M$ - have been sufficiently analyzed in [2]. As stated, the exact dependence which the Green function in [2] has on the radial variable is unknown. By contrast, the dependence of the Green function in (6) on space-time is explicit. Therefore an appropriate analytical extension of (6) to values of $D\left(x_{2}-x_{1}\right)$ corresponding to $0<r \leq 2 M$ is expected to yield a welldefined and unique propagator in the interior region of the Schwarzschild black hole. Such a propagator will identically satisfy two central demands. These are, the demand for the correct divergence structure at the coincidence space-time limit $x_{2} \rightarrow x_{1}$ and the demand for regularity away from that limit. The result of the analytical extension of (6) must, in the interior of the Schwarzschild black hole, define a Green function whose singularity structure will correspond to a quadratic divergence and which, away from the coincidence space-time limit, will be a regular (that is, single valued and analytic) function of space-time for all values of the space-time coordinates in its domain. In what follows the appropriate analytical extension of (6) will, indeed, yield such a Green function.

\section{The Analytical Extension - Singular Part}

The singular part $D_{a s}\left(x_{2}-x_{1}\right)$ of the Green function in (6) - that is, the part which is singular at $x_{2} \rightarrow x_{1}$ and coincides with the asymptotic expression of that Green function [4] - is

$$
D_{a s}\left(x_{2}-x_{1}\right)=
$$

$\frac{2}{\beta} \frac{1}{\sqrt{\rho_{1} \rho_{2}}} \sum_{l=0}^{\infty} \sum_{m=-l}^{l} Y_{l m}\left(\theta_{2}, \phi_{2}\right) Y_{l m}^{*}\left(\theta_{1}, \phi_{1}\right) \sum_{p=0}^{\infty} e^{i \frac{p}{\beta}\left(\tau_{2}-\tau_{1}\right)} \int_{u_{0}[p]}^{\infty} d u \frac{\cos \left[\frac{\pi}{4 \beta}(4 u+2 p+3)\left(\rho_{2}-\rho_{1}\right)\right]}{\pi^{2} u^{2}+4\left(l^{2}+l+1\right)}$

the radial-temporal sector of which is the sum of

$$
\frac{e^{i \frac{3 \pi}{4 \beta}\left(\rho_{2}-\rho_{1}\right)}}{\beta \sqrt{\rho_{1} \rho_{2}}} \sum_{p=0}^{\infty} e^{i \frac{p}{\beta}\left(\tau_{2}-\tau_{1}\right)} e^{i \frac{\pi}{2 \beta} p\left(\rho_{2}-\rho_{1}\right)} \int_{u_{0}[p]}^{\infty} d u \frac{e^{i \frac{\pi}{\beta} u\left(\rho_{2}-\rho_{1}\right)}}{\pi^{2} u^{2}+4\left(l^{2}+l+1\right)}
$$

and

$$
\frac{e^{-i \frac{3 \pi}{4 \beta}\left(\rho_{2}-\rho_{1}\right)}}{\beta \sqrt{\rho_{1} \rho_{2}}} \sum_{p=0}^{\infty} e^{i \frac{p}{\beta}\left(\tau_{2}-\tau_{1}\right)} e^{-i \frac{\pi}{2 \beta} p\left(\rho_{2}-\rho_{1}\right)} \int_{u_{0}[p]}^{\infty} d u \frac{e^{-i \frac{\pi}{\beta} u\left(\rho_{2}-\rho_{1}\right)}}{\pi^{2} u^{2}+4\left(l^{2}+l+1\right)}
$$


These two expressions are, of course, valid in the exterior region of the Schwarzschild black-hole space-time - that is, in the region defined by $r \geq 2 M$ and, for that matter, by $\rho \geq 0$. The naive replacement of that range in these two expressions by $0 \leq r<2 M$ with its concomitant imaginary values of $\rho$ causes either one of them to be sharply divergent for arbitrary space-time separations in the interior region. Such a replacement is, consequently, unacceptable. Clearly, what is required in the interior is a mathematical expression for the propagator the radial sector of which will exclusively feature purely imaginary values of $\rho$ while remaining convergent away from the coincidence space-time limit $x_{2} \rightarrow x_{1}$. In what follows, both (11) and (12) will be respectively subjected to a specific analytical extension which will be shown to correspond identically to the analytical extension of (11) and (12) in the interior region of the black hole. In both (11) and (12) the assumption is that $\rho_{2}-\rho_{1}>0$ and $\tau_{2}-\tau_{1}>0$. The analytical extension will first be applied to (11).

Upon extending $u \in R$ to $\tilde{u} \in C$ the function which is integrated over $u$ develops poles at $\pm i \frac{\sqrt{4\left(l^{2}+l+1\right)}}{\pi}$. Hence, over the closed contour $c_{\tilde{u}}$ of Fig. 1 - specified by the segments (i) $u-u_{0}>0$ (ii) $\left|\tilde{u}-u_{0}\right| e^{i \theta_{\tilde{u}}^{\prime}}, 0 \leq \theta_{\tilde{u}}^{\prime} \leq \frac{\pi}{2},|\tilde{u}|=u$, and (iii) $\left|\tilde{u}-u_{0}\right| e^{i \frac{\pi}{2}}-$ by Cauchy's theorem it is

$$
\oint_{c_{\tilde{u}}} d \tilde{u} \frac{e^{i \frac{\pi}{\beta} \tilde{u}\left(\rho_{2}-\rho_{1}\right)}}{\pi^{2} \tilde{u}^{2}+4\left(l^{2}+l+1\right)}=0
$$

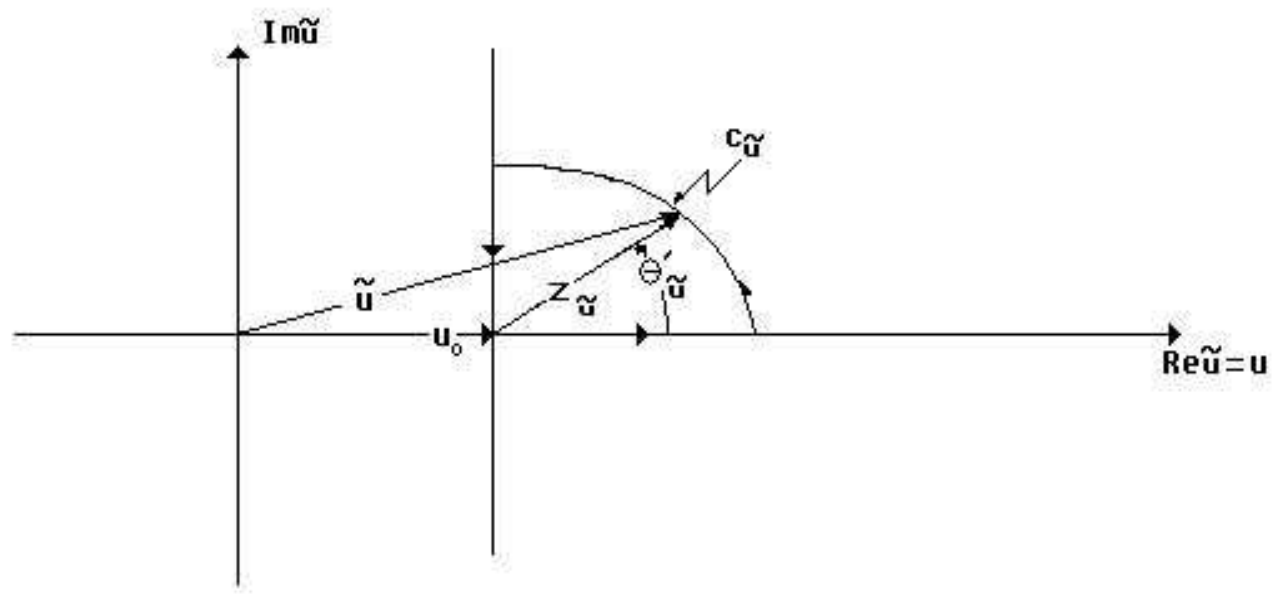

FIGURE 1. Integration over the infinite contour $c_{\tilde{u}}$ in the $\tilde{u}$-complex plane. The choice of $c_{\tilde{u}}$ is dictated by the demand for vanishing contribution along the infinite quadrant.

Setting $\tilde{u}-u_{0}=z_{\tilde{u}}$ it is on the large quadrant $c_{\tilde{u}}^{\theta^{\prime}}$ of $c_{\tilde{u}}$ 


$$
\begin{gathered}
I_{c_{\tilde{u}}^{\theta^{\prime}}}=\int_{c_{\tilde{u}}^{\theta^{\prime}}} d \tilde{u} \frac{e^{i \frac{\pi}{\beta} \tilde{u}\left(\rho_{2}-\rho_{1}\right)}}{\pi^{2} \tilde{u}^{2}+4\left(l^{2}+l+1\right)}=e^{i \frac{\pi}{\beta} u_{0}[p]\left(\rho_{2}-\rho_{1}\right)} \int_{c_{\tilde{u}}^{\theta^{\prime}}} d z_{\tilde{u}} \frac{e^{i \frac{\pi}{\beta} z_{\tilde{u}}\left(\rho_{2}-\rho_{1}\right)}}{\pi^{2}\left(z_{\tilde{u}}+u_{0}\right)^{2}+4\left(l^{2}+l+1\right)}= \\
i e^{i \frac{\pi}{\beta} u_{0}[p]\left(\rho_{2}-\rho_{1}\right)} \int_{0}^{\frac{\pi}{2}} d \theta_{\tilde{u}}^{\prime}\left|z_{\tilde{u}}\right| e^{i \theta_{\tilde{u}}^{\prime}} \frac{e^{i \frac{\pi}{\beta}\left|z_{\tilde{u}}\right| \cos \theta_{\tilde{u}}^{\prime}\left(\rho_{2}-\rho_{1}\right)} e^{-\frac{\pi}{\beta}\left|z_{\tilde{u}}\right| \sin \theta_{\tilde{u}}^{\prime}\left(\rho_{2}-\rho_{1}\right)}}{\pi^{2}\left(z_{\tilde{u}}+u_{0}\right)^{2}+4\left(l^{2}+l+1\right)}
\end{gathered}
$$

so that letting $|\tilde{u}| \rightarrow \infty$ while keeping $u_{0}$ at a fixed value it is

$$
\lim _{\left|z_{\tilde{u}}\right| \rightarrow \infty} I_{c_{\tilde{u}}^{\theta^{\prime}}}=0
$$

The contribution along the stated third segment of the infinite countour $c_{\tilde{u}}$ is

$I_{c_{\tilde{u}}^{\pi / 2}}=\int_{u_{0}+i \infty}^{u_{0}[p]} d \tilde{u} \frac{e^{i \frac{\pi}{\beta} \tilde{u}\left(\rho_{2}-\rho_{1}\right)}}{\pi^{2} \tilde{u}^{2}+4\left(l^{2}+l+1\right)}=e^{i \frac{\pi}{\beta} u_{0}[p]\left(\rho_{2}-\rho_{1}\right)} \int_{i \infty}^{0} d z_{\tilde{u}} \frac{e^{i \frac{\pi}{\beta} z_{\tilde{u}}\left(\rho_{2}-\rho_{1}\right)}}{\pi^{2}\left(z_{\tilde{u}}+u_{0}\right)^{2}+4\left(l^{2}+l+1\right)}$

with $\left|z_{\tilde{u}}\right|=w$ it is

$$
I_{c_{\tilde{u}}^{\pi / 2}}=e^{i \frac{\pi}{\beta} u_{0}[p]\left(\rho_{2}-\rho_{1}\right)} \int_{\infty}^{0} d w e^{i \frac{\pi}{2}} \frac{e^{-\frac{\pi}{\beta} w\left(\rho_{2}-\rho_{1}\right)}}{\pi^{2}\left(u_{0}^{2}-w^{2}\right)+4\left(l^{2}+l+1\right)+2 i \pi^{2} u_{0} w}
$$

As a consequence of (14) and (15) the statement in (13) implies

$$
\int_{u_{0}[p]}^{\infty} d u \frac{e^{i \frac{\pi}{\beta} u\left(\rho_{2}-\rho_{1}\right)}}{\pi^{2} u^{2}+4\left(l^{2}+l+1\right)}=i e^{i \frac{\pi}{\beta} u_{0}[p]\left(\rho_{2}-\rho_{1}\right)} \int_{0}^{\infty} d w \frac{e^{-\frac{\pi}{\beta} w\left(\rho_{2}-\rho_{1}\right)}}{\pi^{2}\left(u_{0}^{2}-w^{2}\right)+4\left(l^{2}+l+1\right)+2 i \pi^{2} u_{0} w}
$$

In view of this result (11) becomes

$$
\begin{aligned}
& i \frac{e^{i \frac{3 \pi}{4 \beta}\left(\rho_{2}-\rho_{1}\right)}}{\beta \sqrt{\rho_{1} \rho_{2}}} \sum_{p=0}^{\infty} e^{i \frac{p}{\beta}\left(\tau_{2}-\tau_{1}\right)} e^{i \frac{\pi}{2 \beta} p\left(\rho_{2}-\rho_{1}\right)} e^{i \frac{\pi}{\beta} u_{0}[p]\left(\rho_{2}-\rho_{1}\right)} \times \\
& \int_{0}^{\infty} d w \frac{e^{-\frac{\pi}{\beta} w\left(\rho_{2}-\rho_{1}\right)}}{\pi^{2}\left(u_{0}^{2}[p]-w^{2}\right)+4\left(l^{2}+l+1\right)+2 i \pi^{2} u_{0}[p] w}
\end{aligned}
$$

In order to advance the analytical extension of (11) it is necessary to convert the infinite series over $p$ in (17) to a contour integral. Since, upon extending $p \in R$ to $\tilde{p} \in C$, the function of $\tilde{p}$ which emerges from (17) tends to zero at $|\tilde{p}| \rightarrow \infty$ faster than $|\tilde{p}|^{-1}$ the residue theorem implies that (17) can be expressed as

$$
\begin{gathered}
\frac{i}{\beta} \frac{1}{2 i} \oint_{c_{\tilde{p}}} d \tilde{p} \frac{(-1)^{\tilde{p}}}{\sin (\pi \tilde{p})} e^{i \frac{\tilde{p}}{\beta}\left(\tau_{2}-\tau_{1}\right)} e^{i \frac{\pi}{2 \beta} \tilde{p}\left(\rho_{2}-\rho_{1}\right)} e^{i \frac{\pi}{\beta} u_{0}[\tilde{p}]\left(\rho_{2}-\rho_{1}\right)} \frac{e^{i \frac{3 \pi}{4 \beta}\left(\rho_{2}-\rho_{1}\right)}}{\sqrt{\rho_{1} \rho_{2}}} \times \\
\int_{0}^{\infty} d w \frac{e^{-\frac{\pi}{\beta} w\left(\rho_{2}-\rho_{1}\right)}}{\pi^{2}\left(u_{0}^{2}[\tilde{p}]-w^{2}\right)+4\left(l^{2}+l+1\right)+2 i \pi^{2} u_{0}[\tilde{p}] w}
\end{gathered}
$$


on condition that the infinite contour $c_{\tilde{p}}$ in the $\tilde{p}$-complex plane encompasses all nonnegative integers, as in Fig.2. The reason the factor $\frac{e^{i \frac{3 \pi}{4 \beta}\left(\rho_{2}-\rho_{1}\right)}}{\sqrt{\rho_{1} \rho_{2}}}$ appears in the integrand will be elucidated in what follows.

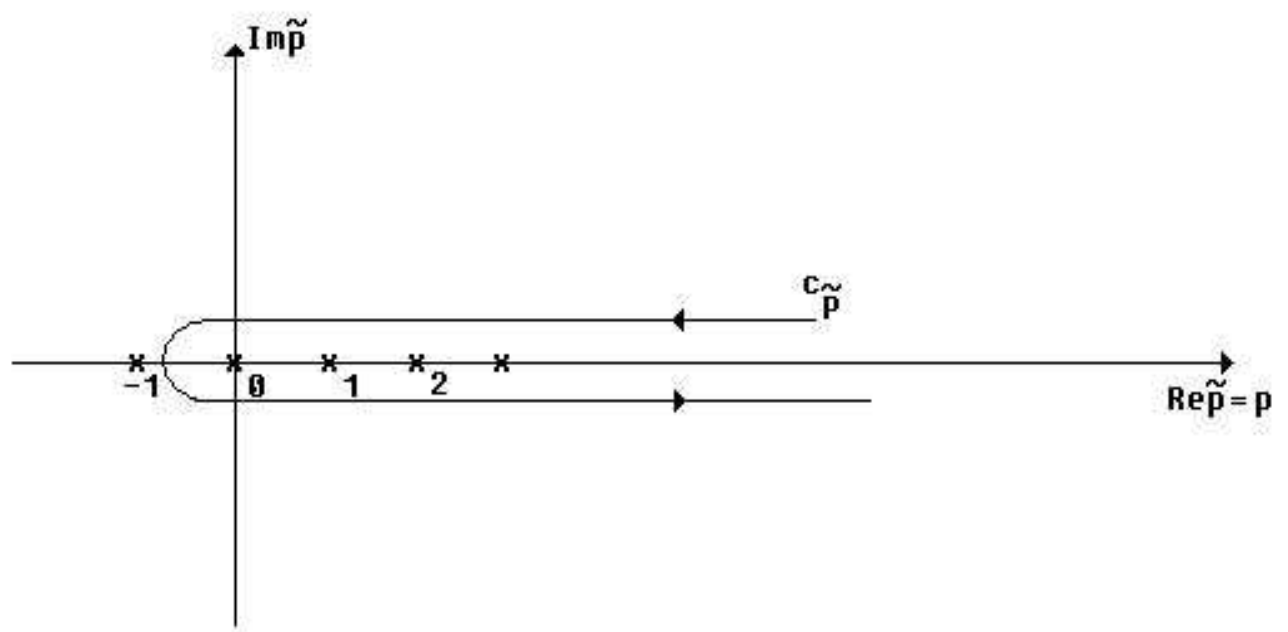

Figure 2. An infinite contour for the integral in (18)

Attention is invited to the fact that since the expression $\frac{\tau_{2}-\tau_{1}}{\beta}$ is an angular coordinate the presence of $\tilde{p} \in C$ in the corresponding exponential in (18) imposes the physical requirement that the evaluation of the contour integral in the latter yield a single-valued function of the temporal difference $\tau_{2}-\tau_{1}$. That this is, indeed, the case follows from the character of (18) as an integral representation of (11).

The integral in (18) taken along the contour of Fig.2 can not, in any physically meaningful way, be associated with the imaginary values of $\rho$ stemming from (2) for $r<2 M$. The primary reason is that, on general theoretical grounds, any analytical extension in transfer space necessarily induces a corresponding extension in the coordinate variables which precludes the arbitrary replacement of $\rho$ by $\pm i|\rho|$. In addition, the arbitrary replacement of $\rho$ by $-i|\rho|$ in (18) would generate an oscillatory integrand of a sharply divergent amplitude at $|\tilde{p}| \rightarrow \infty$ 3. However, the merit of (18) lies in the fact that the contour $c_{\tilde{p}}$ in Fig. 2 can be properly deformed so as to yield a convergent expression in the interior of the black hole. Since integration in (18) along any contour which includes identically the same infinite set of poles as that in Fig.2 results invariably in (17) the contour in Fig. 2 can be deformed to one which (i) avoids each pole through an arbitrarily small semi-circle just below the real $p$-axis, (ii) extends the path of integration in the $\tilde{p}$-complex plane from the real axis to the imaginary axis through a large segment of

\footnotetext{
${ }^{3}$ The arbitrary replacement $\rho \rightarrow+i|\rho|$ would, likewise, generate the same effect to the corresponding analytical extension of (12).
} 
constant radius and of positive ("counterclockwise") orientation and (iii) closes the path of integration along the imaginary axis from positive imaginary infinity to the circumference of a circular segment of vanishing radius about zero. The corresponding contour $c_{\tilde{p}}^{\prime}$ is depicted in Fig.3.

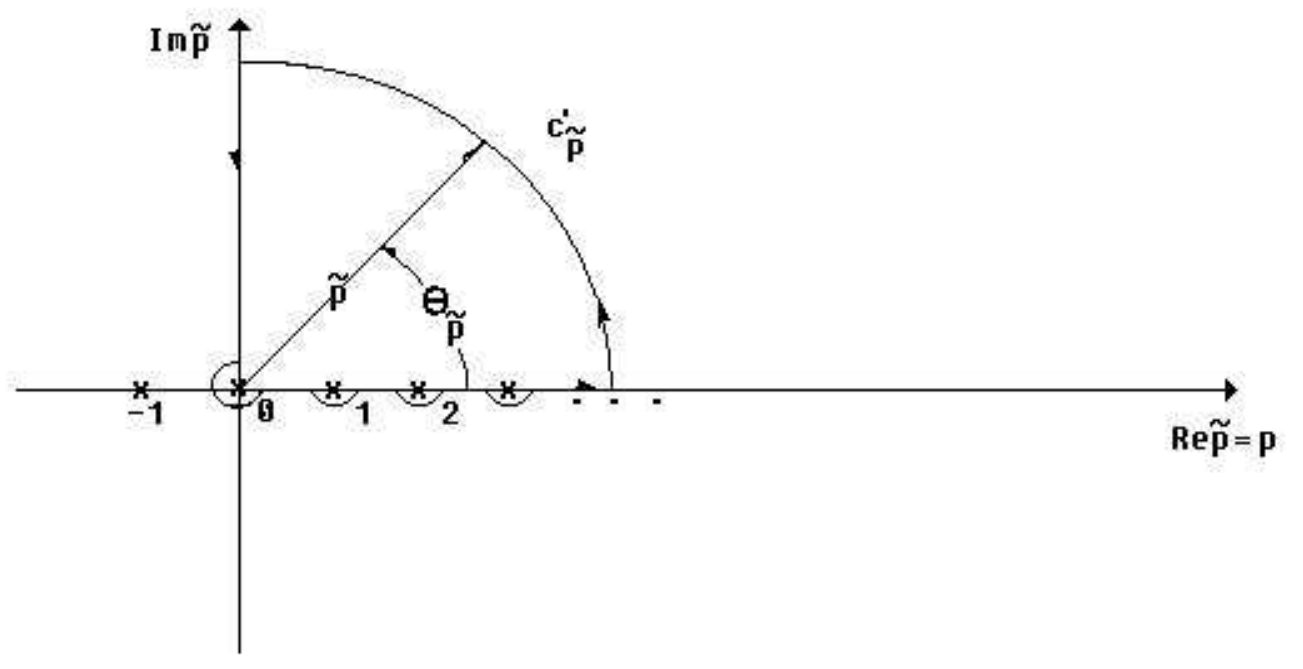

FiguRE 3. An equivalent infinite contour for the integral in (18)

The objective of a convergent expression for the propagator in the interior region necessitates a careful use of the rotation $p \rightarrow|\tilde{p}| e^{i \theta_{\tilde{p}}}$ along the stated contour in Fig.3. First of all, since in view of (6) it is $u_{0}[p]>>p$ the stated rotation implies that $u_{0}[|\tilde{p}|]>>$ $|\tilde{p}|$. At once, this condition is valid for each $\theta_{\tilde{p}} \in\left[0, \frac{\pi}{2}\right]$ in the $\tilde{p}$-complex plane since the integration along the contour $c_{\tilde{u}}$ which eventuated in (16) has been performed in the $\tilde{u}$ complex plane and is, for that matter, irrelevant to $\theta_{\tilde{p}}$. In effect, the rotation $p \rightarrow|\tilde{p}| e^{i \theta_{\tilde{p}}}$ along the contour $c_{\tilde{p}}^{\prime}$ implies that

$$
u_{0}[p] \rightarrow\left|u_{0}[\tilde{p}]\right| e^{i \theta_{\tilde{p}}} ;\left|u_{0}[\tilde{p}]\right|=u_{0}[|\tilde{p}|]>>|\tilde{p}|
$$

In addition, since - as stated - the analytical extension $p \rightarrow|\tilde{p}| e^{i \theta_{\tilde{p}}}$ must be offset by a corresponding extension in the associated coordinate variables it is necessary that the integration along the contour $c_{\tilde{p}}^{\prime}$ in Fig. 3 be advanced in the context of the simultaneous substitutions

$$
\tau_{2}=e^{-i \theta_{\tilde{p}}} \xi_{2} ; \tau_{1}=e^{-i \theta_{\tilde{p}}} \xi_{1} ; \xi_{2}, \xi_{1} \in R
$$

and

$$
\rho_{2}=e^{-i \theta_{\tilde{p}}} \zeta_{2} ; \rho_{1}=e^{-i \theta_{\tilde{p}}} \zeta_{1} ; \zeta_{2}, \zeta_{1} \in R
$$


The new variables $\xi_{i}$ and $\zeta_{i} ; i=1,2$ being the corresponding magnitudes of $\tau_{i}$ and $\rho_{i}$ respectively coincide with the latter in the exterior region of the black-hole geometry. The physical significance of this fact will be discussed in the final result. It is stressed, however, that (21) reflects a general property of the radial variable $\rho$ in the event that $p \rightarrow$ $|\tilde{p}| e^{i \theta_{\tilde{p}}}$. Consequently, the factor $\frac{e^{i \frac{3 \pi}{4 \beta}\left(\rho_{2}-\rho_{1}\right)}}{\sqrt{\rho_{1} \rho_{2}}}$ - which in (11) is $p$-independent - develops, as a result of (21), an implicit dependence on $\tilde{p}$ and must be included in the contour integral. This is explicitly the case in (18).

Deforming the contour $c_{\tilde{p}}$ to $c_{\tilde{p}}^{\prime}$ and replacing (19), (20) and (21) in the integral over $\tilde{p}$ featured in (18), now taken over the large quadrant of $c_{\tilde{p}}^{\prime}$, results in

$$
\begin{aligned}
I_{c_{\tilde{p}}^{\prime}}^{\theta_{\tilde{p}}}=i|\tilde{p}| & \int_{0}^{\frac{\pi}{2}} d \theta_{\tilde{p}} e^{i \theta_{\tilde{p}}} \frac{(-1)^{\tilde{p}}}{\sin (\pi \tilde{p})} e^{i \frac{|\tilde{p}|}{\beta}\left(\xi_{2}-\xi_{1}\right)} e^{i \frac{\pi}{2 \beta}|\tilde{p}|\left(\zeta_{2}-\zeta_{1}\right)} e^{i \frac{\pi}{\beta} u_{0}[|\tilde{p}|]\left(\zeta_{2}-\zeta_{1}\right)} \frac{e^{i \frac{3 \pi}{4 \beta} e^{-i \theta_{\tilde{p}}\left(\zeta_{2}-\zeta_{1}\right)}}}{e^{-i \theta_{\tilde{p}}} \sqrt{\zeta_{1} \zeta_{2}}} \times \\
& \int_{0}^{\infty} d w \frac{e^{-\frac{\pi}{\beta} w e^{-i \theta_{\tilde{p}}}\left(\zeta_{2}-\zeta_{1}\right)}}{\pi^{2}\left(u_{0}^{2}[|\tilde{p}|] e^{i 2 \theta_{\tilde{p}}}-w^{2}\right)+4\left(l^{2}+l+1\right)+2 i \pi^{2} u_{0}[|\tilde{p}|] e^{i \theta_{\tilde{p}}} w}
\end{aligned}
$$

It can be seen at this stage that (11) admits, in principle, several extensions depending on the manner in which $(-1)^{\tilde{p}}$ is expressed. Apparently, certain conditions are necessary in order to select a unique, physically valid analytical extension. To that effect it must be observed that the Green function in (6) features, in its singular part at the coincidence space-time limit $x_{2} \rightarrow x_{1}$, a logarithmic divergence in $1+1$-dimensions and a quadratic divergence in four dimensions [4]. Such a divergence structure identically characterises also (11) and (12). It is imperative, for that matter, that the same divergence structure characterise the analytical extension of (11) and (12) independently since that extension is, essentially, an integral representation of (11) and (12) respectively. In addition, the stated divergence structure is, on general theoretical grounds [4], also expected in the interior of the black hole - on condition that the Schwarzschild radial coordinates associated with the Feynman propagator receive values well above the $r=0$, singularity-related value. As a consequence, any analytical extension of these two expressions which does not preserve their stated divergence structure must be ruled out. At once, consistency with this requirement imposes the additional demand that the stated divergence structure be preserved at each stage of the analytical extension of (11) and, independently, of (12).

As a consequence of this demand it is, in $(22),(-1)^{\tilde{p}}=\left(e^{-i \pi}\right)^{\tilde{p}}$ so that

$$
\begin{gathered}
\frac{(-1)^{\tilde{p}}}{\sin (\pi \tilde{p})}= \\
e^{-i \pi|\tilde{p}| \cos \theta_{\tilde{p}}} \frac{2 e^{\pi|\tilde{p}| s i n \theta_{\tilde{p}}}}{\sin \left(\pi|\tilde{p}| \cos \theta_{\tilde{p}}\right)\left(e^{\pi|\tilde{p}| \sin \theta_{\tilde{p}}}+e^{-\pi|\tilde{p}| \sin \theta_{\tilde{p}}}\right)+i \cos \left(\pi|\tilde{p}| \cos \theta_{\tilde{p}}\right)\left(e^{\pi|\tilde{p}| \sin \theta_{\tilde{p}}}-e^{-\pi|\tilde{p}| \sin \theta_{\tilde{p}}}\right)}
\end{gathered}
$$

The right side of this relation can, for large values of $|\tilde{p}|$, be approximated by

$$
e^{-i \pi|\tilde{p}| \cos \theta_{\tilde{p}}} \frac{2 e^{\pi|\tilde{p}| \sin \theta_{\tilde{p}}}}{\left[\sin \left(\pi|\tilde{p}| \cos \theta_{\tilde{p}}\right)+i \cos \left(\pi|\tilde{p}| \cos \theta_{\tilde{p}}\right)\right] e^{\pi|\tilde{p}| \sin \theta_{\tilde{p}}}}=-2 i
$$


in view of the fact that along the quadrant of $c_{\tilde{p}}^{\prime}$ it is $0 \leq \sin \theta_{\tilde{p}} \leq 1$.

Replacing this result in $(22)$ it is seen that as $|\tilde{p}| \rightarrow \infty$ the expression $I_{c_{\tilde{p}}^{\prime}}^{\theta_{\tilde{p}}}$ is purely oscillatory in the ultra-violet domain of the integral over $w$, if $x_{2} \neq x_{1}$. In order to ensure a vanishing result at $|\tilde{p}| \rightarrow \infty$, for that matter, it is also necessary to implement the substitution

$$
\xi_{2}-\xi_{1} \rightarrow\left(\xi_{2}-\xi_{1}\right)+i v ; 0<v<<1 ; x_{2} \neq x_{1} ; \theta_{\tilde{p}} \in\left(0, \frac{\pi}{2}\right)
$$

on the understanding that the limit $v \rightarrow 0$ is implicit at the end of the calculation in this range of $\theta_{\tilde{p}}$ and, independently, at $x_{2} \rightarrow x_{1}$ prior to taking $|\tilde{p}| \rightarrow \infty$.

In effect, it is

$$
\lim _{|\tilde{p}| \rightarrow \infty} I_{c_{\tilde{p}}^{\prime}}^{\theta_{\tilde{p}}}=0 ; x_{2} \neq x_{1}
$$

At once, it can be seen that taking the coincidence space-time limit $x_{2} \rightarrow x_{1}$ prior to taking $|\tilde{p}| \rightarrow \infty$ results in the desired logarithmic divergence, stated above. In this respect it can readily be confirmed that the replacement $(-1)^{\tilde{p}}=\left(e^{i \pi}\right)^{\tilde{p}}$ is inadmissible in (22) as, at $|\tilde{p}| \rightarrow \infty$, it would yield a vanishing result also for $x_{2} \rightarrow x_{1}$.

The integral over $\tilde{p}$ in (18), now taken along that segment of $c_{\tilde{p}}^{\prime}$ which lies on the imaginary axis, corresponds to $\theta_{\tilde{p}}=\frac{\pi}{2}$. Again, in view of (19), (20) and (21) that integral is

$$
\begin{aligned}
I_{c_{\tilde{p}}^{\prime}}^{\frac{\pi}{2}}=\int_{\infty}^{\epsilon \rightarrow 0} d|\tilde{p}| i \frac{(-1)^{i|\tilde{p}|}}{i \sinh (\pi|\tilde{p}|)} e^{i \frac{|\tilde{p}|}{\beta}\left(\xi_{2}-\xi_{1}\right)} e^{i \frac{\pi}{2 \beta}|\tilde{p}|\left(\zeta_{2}-\zeta_{1}\right)} e^{i \frac{\pi}{\beta} u_{0}[|\tilde{p}|]\left(\zeta_{2}-\zeta_{1}\right)} \frac{e^{i \frac{3 \pi}{4 \beta}(-i)\left(\zeta_{2}-\zeta_{1}\right)}}{(-i) \sqrt{\zeta_{1} \zeta_{2}}} \times \\
\\
\int_{0}^{\infty} d w \frac{e^{-\frac{\pi}{\beta} w(-i)\left(\zeta_{2}-\zeta_{1}\right)}}{-\pi^{2}\left(u_{0}^{2}[|\tilde{p}|]+w^{2}\right)+4\left(l^{2}+l+1\right)-2 \pi^{2} u_{0}[|\tilde{p}|] w}
\end{aligned}
$$

where - as is evident in Fig. $3-\epsilon<<1$ corresponds to the radius of the circular segment centred at zero.

Replacing

$$
\frac{(-1)^{i|\tilde{p}|}}{\sinh (\pi|\tilde{p}|)}=2 \frac{e^{\pi|\tilde{p}|}}{e^{\pi|\tilde{p}|}-e^{-\pi|\tilde{p}|}}=\frac{2}{1-e^{-2 \pi|\tilde{p}|}}
$$

in (24) yields

$$
\begin{aligned}
I_{c_{\tilde{p}}^{\prime}}^{\frac{\pi}{2}}=2 i \int_{\infty}^{\epsilon \rightarrow 0} d|\tilde{p}| \frac{1}{1-e^{-2 \pi|\tilde{p}|}} e^{i \frac{i \tilde{p} \mid}{\beta}\left(\xi_{2}-\xi_{1}\right)} e^{i \frac{\pi}{2 \beta}|\tilde{p}|\left(\zeta_{2}-\zeta_{1}\right)} e^{i \frac{\pi}{\beta} u_{0}[|\tilde{p}|]\left(\zeta_{2}-\zeta_{1}\right)} \frac{e^{\frac{3 \pi}{4 \beta}\left(\zeta_{2}-\zeta_{1}\right)}}{\sqrt{\zeta_{1} \zeta_{2}}} \times \\
\int_{0}^{\infty} d w \frac{e^{i \frac{\pi}{\beta} w\left(\zeta_{2}-\zeta_{1}\right)}}{-\pi^{2}\left(u_{0}^{2}[|\tilde{p}|]+w^{2}\right)+4\left(l^{2}+l+1\right)-2 \pi^{2} u_{0}[|\tilde{p}|] w}
\end{aligned}
$$

Again, at $x_{2} \rightarrow x_{1}$ this expression manifestly features the desired logarithmic divergence. If, instead, the analytical extension were based on $(-1)^{\tilde{p}}=\left(e^{i \pi}\right)^{\tilde{p}}$ then $(25)$ would vanish at $x_{2} \rightarrow x_{1}$. 
In what follows the segment of the contour $c_{\tilde{p}}^{\prime}$ in Fig.3 which extends along Re $\tilde{p}$ obviating each pole other than $\tilde{p}=0$ through an arbitrarily small semicircle and the pole at $\tilde{p}=0$ through an arbitrarily small circular quadrant will be denoted by $c_{\tilde{p}}^{+}$. For notational convenience the corresponding Cauchy principal value, that is the integral over $\tilde{p}$ in (18) evaluated along $c_{\tilde{p}}^{+}$, will be denoted by $I_{c_{\tilde{p}}^{\prime}}^{+}$. As a result of (23) it follows that the integral over $\tilde{p}$ in (18) taken along the infinite contour $c_{\tilde{p}}^{\prime}$ is equal to $I_{c_{\tilde{p}}^{\prime}}^{+}+I_{c_{\tilde{p}}^{\prime}}^{\frac{\pi}{2}}+I_{c_{\tilde{p}}^{\prime}}^{s c}$, where $I_{c_{\tilde{p}}^{\prime}}^{s c}$ is the contribution along the small semi-circle centred at zero and extending from $\theta_{\tilde{p}}=\frac{\pi}{2}$ to $\theta_{\tilde{p}}=\frac{3 \pi}{2}$ in Fig.3. In order to evaluate $I_{c_{\tilde{p}}^{\prime}}^{+}$the integral over $\tilde{p}$ in (18) will now be evaluated along the closed contour $\tilde{c}_{\tilde{p}}$ which (i) extends along $c_{\tilde{p}}^{+}$, (ii) extends the path of integration in the $\tilde{p}$-complex plane from the real axis to the imaginary axis through a large segment of constant radius which is, this time, of negative ("clockwise") orientation below the real axis and (iii) closes the path of integration along the imaginary axis from negative imaginary infinity to the circumference of the circular quadrant of vanishing radius about zero. The contour $\tilde{c}_{\tilde{p}}$ is depicted in Fig.4.

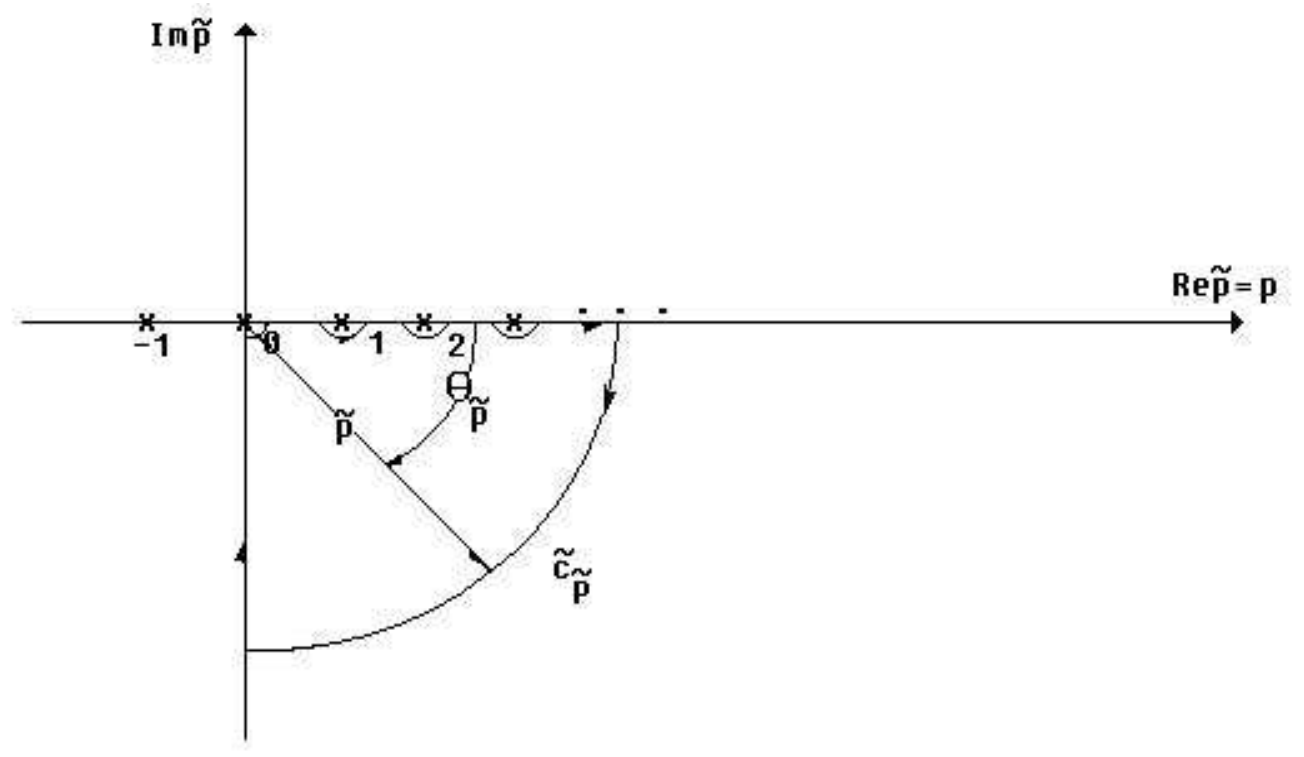

Figure 4. The infinite contour $\tilde{c}_{\tilde{p}}$ in the $\tilde{p}$-complex plane. The pole at $\tilde{p}=0$ is obviated "counterclockwise" through a quadrant of vanishing radius. The horizontal segment of $\tilde{c}_{\tilde{p}}$, including that quadrant, is $c_{\tilde{p}}^{+}$.

Replacing again (19), (20) and (21) in the integral over $\tilde{p}$ featured in (18), now taken over the large quadrant of $\tilde{c}_{\tilde{p}}$, results in

$$
I_{\tilde{c}_{\tilde{p}}}^{\theta_{\tilde{p}}}=i|p| \int_{0}^{-\frac{\pi}{2}} d \theta_{\tilde{p}} e^{i \theta_{\tilde{p}}} \frac{(-1)^{\tilde{p}}}{\sin (\pi \tilde{p})} e^{i \frac{i \tilde{p} \mid}{\beta}\left(\xi_{2}-\xi_{1}\right)} e^{i \frac{\pi}{2 \beta}|\tilde{p}|\left(\zeta_{2}-\zeta_{1}\right)} e^{i \frac{\pi}{\beta} u_{0}[|\tilde{p}|]\left(\zeta_{2}-\zeta_{1}\right)} \frac{e^{i \frac{3 \pi}{4 \beta} e^{-i \theta_{\tilde{p}}}\left(\zeta_{2}-\zeta_{1}\right)}}{e^{-i \theta_{\tilde{p}}} \sqrt{\zeta_{1} \zeta_{2}}} \times
$$




$$
\int_{0}^{\infty} d w \frac{e^{-\frac{\pi}{\beta} w e^{-i \theta_{\tilde{p}}\left(\zeta_{2}-\zeta_{1}\right)}}}{\pi^{2}\left(u_{0}^{2}[|\tilde{p}|] e^{i 2 \theta_{\tilde{p}}}-w^{2}\right)+4\left(l^{2}+l+1\right)+2 i \pi^{2} u_{0}[|\tilde{p}|] e^{i \theta_{\tilde{p}} w}}
$$

In this context the demand for a logarithmic divergence at each stage of the analytical extension as $x_{2} \rightarrow x_{1}$ implies that $(-1)^{\tilde{p}}=\left(e^{i \pi}\right)^{\tilde{p}}$ so that

$$
\begin{gathered}
\frac{(-1)^{\tilde{p}}}{\sin (\pi \tilde{p})}= \\
e^{i \pi|\tilde{p}| \cos \theta_{\tilde{p}}} \frac{2 e^{-\pi|\tilde{p}| \sin \theta_{\tilde{p}}}}{\sin \left(\pi|\tilde{p}| \cos \theta_{\tilde{p}}\right)\left(e^{\pi|\tilde{p}| \sin \theta_{\tilde{p}}}+e^{-\pi|\tilde{p}| \sin \theta_{\tilde{p}}}\right)+i \cos \left(\pi|\tilde{p}| \cos \theta_{\tilde{p}}\right)\left(e^{\pi|\tilde{p}| \sin \theta_{\tilde{p}}}-e^{-\pi|\tilde{p}| \sin \theta_{\tilde{p}}}\right)}
\end{gathered}
$$

The right side of this relation can, for large values of $|\tilde{p}|$, be approximated by

$$
e^{i \pi|\tilde{p}| \cos \theta_{\tilde{p}}} \frac{2 e^{-\pi|\tilde{p}| \sin \theta_{\tilde{p}}}}{\left[\sin \left(\pi|\tilde{p}| \cos \theta_{\tilde{p}}\right)-i \cos \left(\pi|\tilde{p}| \cos \theta_{\tilde{p}}\right)\right] e^{-\pi|\tilde{p}| \sin \theta_{\tilde{p}}}}=2 i
$$

in view of the fact that along the quadrant of $\tilde{c}_{\tilde{p}}$ it is $-1 \leq \sin \theta_{\tilde{p}} \leq 0$. Replacing this result in (26) and giving, as before, $\xi_{2}-\xi_{1} \neq 0$ a small imaginary part for $-\frac{\pi}{2}<\theta_{\tilde{p}}<0$ yields

$$
\lim _{|\tilde{p}| \rightarrow \infty} I_{\tilde{c}_{\tilde{p}}}^{\theta_{\tilde{p}}}=0 ; x_{2} \neq x_{1}
$$

The integral over $\tilde{p}$ in (18), now taken along the segment of $\tilde{c}_{\tilde{p}}$ which lies on the imaginary axis, corresponds to $\theta_{\tilde{p}}=-\frac{\pi}{2}$. Again, in view of (19), (20) and (21) that integral is

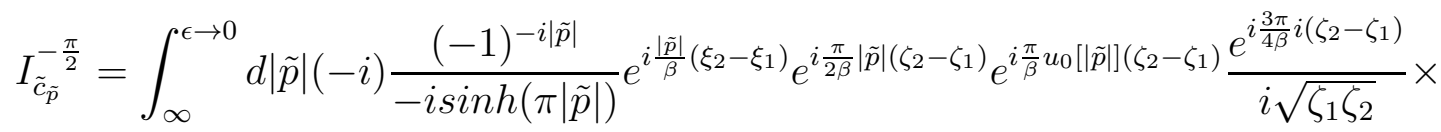

$$
\int_{0}^{\infty} d w \frac{e^{-i \frac{\pi}{\beta} w\left(\zeta_{2}-\zeta_{1}\right)}}{-\pi^{2}\left(u_{0}^{2}[|\tilde{p}|]+w^{2}\right)+4\left(l^{2}+l+1\right)+2 \pi^{2} u_{0}[|\tilde{p}|] w}
$$

where - as is evident in Fig. $4-\epsilon<<1$ corresponds to the radius of the circular quadrant centred at zero.

Replacing

$$
\frac{(-1)^{-i|\tilde{p}|}}{\sinh (\pi|\tilde{p}|)}=2 \frac{e^{\pi|\tilde{p}|}}{e^{\pi|\tilde{p}|}-e^{-\pi|\tilde{p}|}}=\frac{2}{1-e^{-2 \pi|\tilde{p}|}}
$$

in (28) yields

$$
I_{\tilde{c}_{\tilde{p}}}^{-\frac{\pi}{2}}=-2 i \int_{\infty}^{\epsilon \rightarrow 0} d|\tilde{p}| \frac{1}{1-e^{-2 \pi|\tilde{p}|}} e^{i \frac{|\tilde{p}|}{\beta}\left(\xi_{2}-\xi_{1}\right)} e^{i \frac{\pi}{2 \beta}|\tilde{p}|\left(\zeta_{2}-\zeta_{1}\right)} e^{i \frac{\pi}{\beta} u_{0}[|\tilde{p}|]\left(\zeta_{2}-\zeta_{1}\right)} \frac{e^{-\frac{3 \pi}{4 \beta}\left(\zeta_{2}-\zeta_{1}\right)}}{\sqrt{\zeta_{1} \zeta_{2}}} \times
$$




$$
\int_{0}^{\infty} d w \frac{e^{-i \frac{\pi}{\beta} w\left(\zeta_{2}-\zeta_{1}\right)}}{-\pi^{2}\left(u_{0}^{2}[|\tilde{p}|]+w^{2}\right)+4\left(l^{2}+l+1\right)+2 \pi^{2} u_{0}[|\tilde{p}|] w}
$$

At once, since $\tilde{c}_{\tilde{p}}$ in Fig. 4 does not include any poles Cauchy's theorem yields

$$
\begin{gathered}
\oint_{\tilde{c}_{\tilde{p}}} d \tilde{p} \frac{(-1)^{\tilde{p}}}{\sin (\pi \tilde{p})} e^{i \frac{\tilde{p}}{\beta}\left(\tau_{2}-\tau_{1}\right)} e^{i \frac{\pi}{2 \beta} \tilde{p}\left(\rho_{2}-\rho_{1}\right)} e^{i \frac{\pi}{\beta} u_{0}[\tilde{p}]\left(\rho_{2}-\rho_{1}\right)} \frac{e^{i \frac{3 \pi}{4 \beta}\left(\rho_{2}-\rho_{1}\right)}}{\sqrt{\rho_{1} \rho_{2}}} \times \\
\int_{0}^{\infty} d w \frac{e^{-\frac{\pi}{\beta} w\left(\rho_{2}-\rho_{1}\right)}}{\pi^{2}\left(u_{0}^{2}[\tilde{p}]-w^{2}\right)+4\left(l^{2}+l+1\right)+2 i \pi^{2} u_{0}[\tilde{p}] w}=0
\end{gathered}
$$

for the stated integral in (18) taken along that infinite contour. Consequently, in view of $(27),(29)$ and (30) it follows that

$$
\begin{aligned}
I_{c_{\tilde{p}}^{\prime}}^{+}=-2 i & \int_{\epsilon \rightarrow 0}^{\infty} d|\tilde{p}| \frac{1}{1-e^{-2 \pi|\tilde{p}|}} e^{i \frac{|\tilde{p}|}{\beta}\left(\xi_{2}-\xi_{1}\right)} e^{i \frac{\pi}{2 \beta}|\tilde{p}|\left(\zeta_{2}-\zeta_{1}\right)} e^{i \frac{\pi}{\beta} u_{0}[|\tilde{p}|]\left(\zeta_{2}-\zeta_{1}\right)} \frac{e^{-\frac{3 \pi}{4 \beta}\left(\zeta_{2}-\zeta_{1}\right)}}{\sqrt{\zeta_{1} \zeta_{2}}} \times \\
& \int_{0}^{\infty} d w \frac{e^{-i \frac{\pi}{\beta} w\left(\zeta_{2}-\zeta_{1}\right)}}{-\pi^{2}\left(u_{0}^{2}[|\tilde{p}|]+w^{2}\right)+4\left(l^{2}+l+1\right)+2 \pi^{2} u_{0}[|\tilde{p}|] w}
\end{aligned}
$$

Finally, following always the same procedure in the $\tilde{p}$-complex plane it is readily seen that at $|\tilde{p}| \rightarrow 0$ the contribution stemming from the integral along the stated small semi-circle in Fig.3 is 4

$$
\begin{gathered}
I_{c_{\tilde{p}}^{\prime}}^{s c}=\frac{i}{\pi} \frac{1}{\sqrt{\zeta_{1} \zeta_{2}}} \int_{\frac{\pi}{2}}^{\frac{3 \pi}{2}} d \theta e^{i \theta} e^{i \frac{\pi}{\beta}\left[u_{0}[0]+\frac{3}{4} e^{-i \theta}\right]\left(\zeta_{2}-\zeta_{1}\right)} \times \\
\int_{0}^{\infty} d w \frac{e^{-\frac{\pi}{\beta} w e^{-i \theta}\left(\zeta_{2}-\zeta_{1}\right)}}{\pi^{2}\left(u_{0}^{2}[0] e^{2 i \theta}-w^{2}\right)+4\left(l^{2}+l+1\right)+2 i \pi^{2} u_{0}[0] e^{i \theta} w}
\end{gathered}
$$

As stated, the integral over $\tilde{p}$ in (18) taken along the infinite contour $c_{\tilde{p}}^{\prime}$ of Fig.3 is equal to $I_{c_{\tilde{p}}^{\prime}}^{+}+I_{c_{\tilde{p}}^{\prime}}^{\frac{\pi}{2}}+I_{c_{\tilde{p}}^{\prime}}^{s c}$. Hence, as a result of $(31),(25)$ and $(32)$ it is

$$
\begin{gathered}
\frac{1}{2 \beta} \oint_{c_{\tilde{p}}} d \tilde{p} \frac{(-1)^{\tilde{p}}}{\sin (\pi \tilde{p})} e^{i \frac{\tilde{p}}{\beta}\left(\tau_{2}-\tau_{1}\right)} e^{i \frac{\pi}{2 \beta} \tilde{p}\left(\rho_{2}-\rho_{1}\right)} e^{i \frac{\pi}{\beta} u_{0}[\tilde{p}]\left(\rho_{2}-\rho_{1}\right)} \frac{e^{i \frac{3 \pi}{4 \beta}\left(\rho_{2}-\rho_{1}\right)}}{\sqrt{\rho_{1} \rho_{2}}} \times \\
\int_{0}^{\infty} d w \frac{e^{-\frac{\pi}{\beta} w\left(\rho_{2}-\rho_{1}\right)}}{\pi^{2}\left(u_{0}^{2}[\tilde{p}]-w^{2}\right)+4\left(l^{2}+l+1\right)+2 i \pi^{2} u_{0}[\tilde{p}] w}= \\
-\frac{i}{\beta} \frac{e^{-\frac{3 \pi}{4 \beta}\left(\zeta_{2}-\zeta_{1}\right)}}{\sqrt{\zeta_{1} \zeta_{2}}} \int_{\epsilon \rightarrow 0}^{\infty} d|\tilde{p}| \frac{1}{1-e^{-2 \pi|\tilde{p}|}} e^{i \frac{|\tilde{p}|}{\beta}\left(\xi_{2}-\xi_{1}\right)} e^{i \frac{\pi}{\beta}\left[\frac{|\tilde{p}|}{2}+u_{0}[|\tilde{p}|]\right]\left(\zeta_{2}-\zeta_{1}\right)} \times \\
\int_{0}^{\infty} d w \frac{e^{-i \frac{\pi}{\beta} w\left(\zeta_{2}-\zeta_{1}\right)}}{-\pi^{2}\left(u_{0}^{2}[|\tilde{p}|]+w^{2}\right)+4\left(l^{2}+l+1\right)+2 \pi^{2} u_{0}[|\tilde{p}|] w}
\end{gathered}
$$

\footnotetext{
${ }^{4}$ For notational convenience the index $\tilde{p}$ in $\theta_{\tilde{p}}$ will, henceforth, be dropped.
} 


$$
\begin{gathered}
-\frac{i}{\beta} \frac{e^{\frac{3 \pi}{4 \beta}\left(\zeta_{2}-\zeta_{1}\right)}}{\sqrt{\zeta_{1} \zeta_{2}}} \int_{\epsilon \rightarrow 0}^{\infty} d|\tilde{p}| \frac{1}{1-e^{-2 \pi|\tilde{p}|}} e^{i \frac{|\tilde{p}|}{\beta}\left(\xi_{2}-\xi_{1}\right)} e^{i \frac{\pi}{\beta}\left[\frac{|\tilde{p}|}{2}+u_{0}[|\tilde{p}|]\right]\left(\zeta_{2}-\zeta_{1}\right)} \times \\
\int_{0}^{\infty} d w \frac{e^{i \frac{\pi}{\beta} w\left(\zeta_{2}-\zeta_{1}\right)}}{-\pi^{2}\left(u_{0}^{2}[|\tilde{p}|]+w^{2}\right)+4\left(l^{2}+l+1\right)-2 \pi^{2} u_{0}[|\tilde{p}|] w} \\
+\frac{i}{\beta} \frac{1}{2 \pi} \frac{e^{i \frac{\pi}{\beta} u_{0}[0]\left(\zeta_{2}-\zeta_{1}\right)}}{\sqrt{\zeta_{1} \zeta_{2}}} \int_{\frac{\pi}{2}}^{\frac{3 \pi}{2}} d \theta e^{i \theta} e^{i \frac{3 \pi}{4 \beta} e^{-i \theta}\left(\zeta_{2}-\zeta_{1}\right)} \times \\
\int_{0}^{\infty} d w \frac{e^{-\frac{\pi}{\beta} w e^{-i \theta}\left(\zeta_{2}-\zeta_{1}\right)}}{\pi^{2}\left(u_{0}^{2}[0] e^{2 i \theta}-w^{2}\right)+4\left(l^{2}+l+1\right)+2 i \pi^{2} u_{0}[0] e^{i \theta} w}
\end{gathered}
$$

This is the analytical extension of (11). It corresponds to expressing the latter along the imaginary axis of the transfer-space variables $u$ and $p$ respectively. Since $\frac{\xi_{2}-\xi_{1}}{\beta}$ has the same angular significance as $\frac{\tau_{2}-\tau_{1}}{\beta}$ each of the first two terms on the right side of (33) is a multiple-valued function of $\xi_{2}-\xi_{1}$ on account of the presence of $|\tilde{p}| \in R$ in the corresponding exponential. However, as already stated, the sum-total of the three terms on the right side of (33) - being an integral representation of (11) - is always a single-valued function of the temporal coordinate difference $\xi_{2}-\xi_{1}$.

Through (21) the rotation of $p$ to purely imaginary values implies manifestly that $\rho= \pm i\left|\rho_{\text {ext }}\right|$ with $\left|\rho_{\text {ext }}\right|=\zeta$ being the magnitude of the radial variable $\rho_{\text {ext }}$ in the exterior region of the Schwarzschild black-hole geometry - that is, of the radial variable in (2). This result is inherent in (33). At once, (2) implies that for $r \leq 2 M$ the radial variable $\rho_{\text {int }}$ in the interior region satisfies $\rho_{\text {int }}= \pm i\left|\rho_{\text {int }}\right|$. Consequently, the radial variable $\rho$ in (33) is in coincidence with $\rho_{\text {int }}$. This result, namely that

$$
\rho_{\text {int }}= \pm i\left|\rho_{\text {ext }}\right| ;\left|\rho_{\text {int }}\right|=\left|\rho_{\text {ext }}\right|
$$

expresses the fact that the right side of (33) corresponds to an analytical extension of (11) in the interior region of the Schwarzschild black-hole geometry. At once, at the coincidence space-time limit $x_{2} \rightarrow x_{1}$ each of the first two terms on the right side of (33) explicitly features the desired logarithmic divergence while remaining manifestly convergent away from that limit. Although at $x_{2} \rightarrow x_{1}$ the expression in (33) itself is formally indeterminate it is clear that the independent presence of the stated logarithmic divergence in the first two terms signifies a logarithmic divergence for the entire expression in (33). This fact shall be rigorously established in section $\mathrm{V}$ for the entire Green function in four dimensions. Consequently, the expression on the right side of (33) is, indeed, the physically valid analytical extension of (11) in the interior region of the Schwarzschild black hole. The crucial aspect in its derivation has been the demand that the analytical extension be such as to preserve the logarithmic divergence of (11) at each stage of the calculation.

It should also be remarked that, although to each value of $\rho$ in (2) there corresponds a value of $\left|\rho_{\text {int }}\right|$, there is an infinite range of values of $\left|\rho_{\text {int }}\right|$ which do not correspond to any value of $\rho$ in the exterior region. This is readily understood since in the exterior region of the black hole it is $0 \leq \rho \leq \beta$ whereas in the interior region it is $0<\left|\rho_{\text {int }}\right|<\infty$. 
Consequently, in terms of the Schwarzschild radial variable $r$, the maximum possible range of validity of this analytical extension of $D\left(x_{2}-x_{1}\right)$ in the interior region of the black hole is $(0, M)$. Of course, as will be established in due course, the actual range of validity in the interior region is smaller than that since $D\left(x_{2}-x_{1}\right)$ is, in the exterior region, valid for a subset of values of $r \in[0, \infty)$.

The analytical extension of (12) will be obtained through the same procedure so that the analysis leading to the final result will be concise.

Upon extending $u \in R$ to $\tilde{u} \in C$ Cauchy's theorem implies

$$
\oint_{e_{\tilde{u}}} d \tilde{u} \frac{e^{-i \frac{\pi}{\beta} \tilde{u}\left(\rho_{2}-\rho_{1}\right)}}{\pi^{2} \tilde{u}^{2}+4\left(l^{2}+l+1\right)}=0
$$

with $e_{\tilde{u}}$ being the contour in Fig.5.

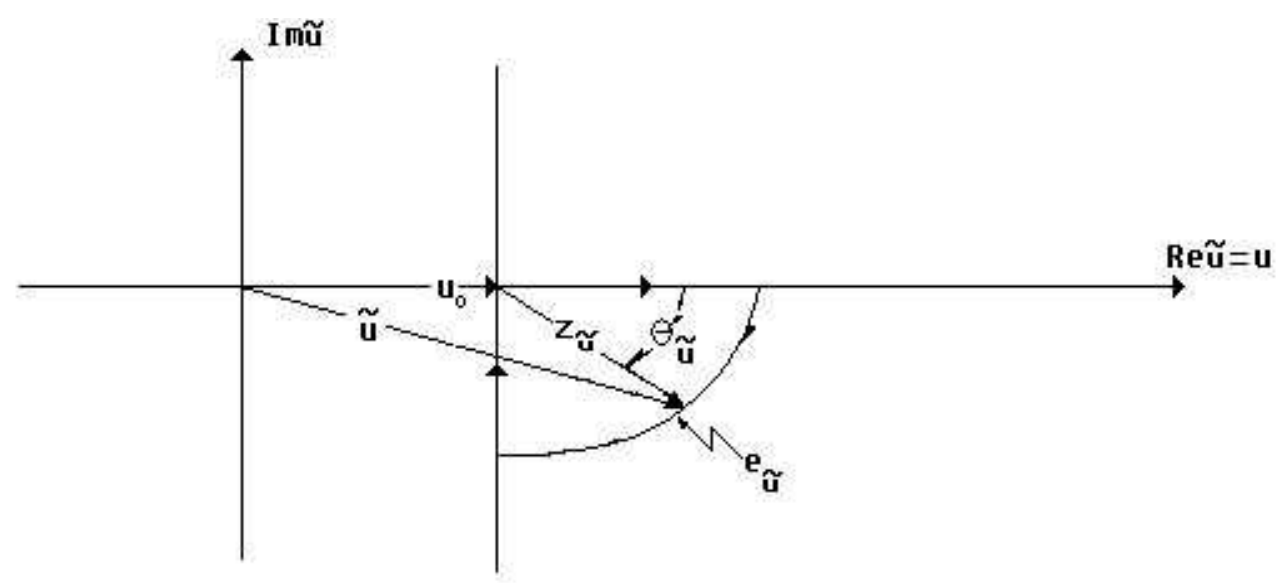

FigURE 5. Integration over the infinite contour $e_{\tilde{u}}$ in the $\tilde{u}$-complex plane. The choice of $e_{\tilde{u}}$ is dictated by the demand for vanishing contribution along the infinite quadrant.

Setting $\tilde{u}-u_{0}=z_{\tilde{u}}$ it is on the large quadrant $e_{\tilde{u}}^{\theta^{\prime}}$ of $e_{\tilde{u}}$

$$
I_{e_{\tilde{u}}^{\theta^{\prime}}}=i e^{-i \frac{\pi}{\beta} u_{0}[p]\left(\rho_{2}-\rho_{1}\right)} \int_{0}^{-\frac{\pi}{2}} d \theta_{\tilde{u}}^{\prime}\left|z_{\tilde{u}}\right| e^{i \theta_{\tilde{u}}^{\prime}} \frac{e^{-i \frac{\pi}{\beta}\left|z_{\tilde{u}}\right| \cos \theta_{\tilde{u}}^{\prime}\left(\rho_{2}-\rho_{1}\right)} e^{\frac{\pi}{\beta}|z \tilde{u}| \sin \theta_{\tilde{u}}^{\prime}\left(\rho_{2}-\rho_{1}\right)}}{\pi^{2}\left(z_{\tilde{u}}+u_{0}\right)^{2}+4\left(l^{2}+l+1\right)}
$$

so that letting $|\tilde{u}| \rightarrow \infty$ while keeping $u_{0}$ at a fixed value it is

$$
\lim _{\left|z_{\tilde{u}}\right| \rightarrow \infty} I_{e_{\tilde{u}}^{\theta^{\prime}}}=0
$$

The contribution along the imaginary segment of the infinite contour $e_{\tilde{u}}$ is 
$I_{e_{\tilde{u}}^{-\pi / 2}}=\int_{u_{0}-i \infty}^{u_{0}[p]} d \tilde{u} \frac{e^{-i \frac{\pi}{\beta} \tilde{u}\left(\rho_{2}-\rho_{1}\right)}}{\pi^{2} \tilde{u}^{2}+4\left(l^{2}+l+1\right)}=e^{-i \frac{\pi}{\beta} u_{0}[p]\left(\rho_{2}-\rho_{1}\right)} \int_{-i \infty}^{0} d z_{\tilde{u}} \frac{e^{-i \frac{\pi}{\beta} z_{\tilde{u}}\left(\rho_{2}-\rho_{1}\right)}}{\pi^{2}\left(z_{\tilde{u}}+u_{0}\right)^{2}+4\left(l^{2}+l+1\right)}$

with $\left|z_{\tilde{u}}\right|=w$ it is

$$
I_{e_{\tilde{u}}^{-\pi / 2}}=e^{-i \frac{\pi}{\beta} u_{0}[p]\left(\rho_{2}-\rho_{1}\right)} \int_{\infty}^{0} d w e^{-i \frac{\pi}{2}} \frac{e^{-\frac{\pi}{\beta} w\left(\rho_{2}-\rho_{1}\right)}}{\pi^{2}\left(u_{0}^{2}-w^{2}\right)+4\left(l^{2}+l+1\right)-2 i \pi^{2} u_{0} w}
$$

As a consequence of (36) and (37) the statement in (35) implies

$$
\int_{u_{0}[p]}^{\infty} d u \frac{e^{-i \frac{\pi}{\beta} u\left(\rho_{2}-\rho_{1}\right)}}{\pi^{2} u^{2}+4\left(l^{2}+l+1\right)}=-i e^{-i \frac{\pi}{\beta} u_{0}[p]\left(\rho_{2}-\rho_{1}\right)} \int_{0}^{\infty} d w \frac{e^{-\frac{\pi}{\beta} w\left(\rho_{2}-\rho_{1}\right)}}{\pi^{2}\left(u_{0}^{2}-w^{2}\right)+4\left(l^{2}+l+1\right)-2 i \pi^{2} u_{0} w}
$$

In view of this result (12) becomes

$$
\begin{gathered}
-i \frac{e^{-i \frac{3 \pi}{4 \beta}\left(\rho_{2}-\rho_{1}\right)}}{\beta \sqrt{\rho_{1} \rho_{2}}} \sum_{p=0}^{\infty} e^{i \frac{p}{\beta}\left(\tau_{2}-\tau_{1}\right)} e^{-i \frac{\pi}{2 \beta} p\left(\rho_{2}-\rho_{1}\right)} e^{-i \frac{\pi}{\beta} u_{0}[p]\left(\rho_{2}-\rho_{1}\right)} \times \\
\int_{0}^{\infty} d w \frac{e^{-\frac{\pi}{\beta} w\left(\rho_{2}-\rho_{1}\right)}}{\pi^{2}\left(u_{0}^{2}[p]-w^{2}\right)+4\left(l^{2}+l+1\right)-2 i \pi^{2} u_{0}[p] w}
\end{gathered}
$$

As was the case with (11) it is necessary to, analytically, extend the transfer-space variable $p$ and convert the infinite series over $p$ in (39) to a contour integral. The residue theorem then implies that (39) can be expressed as

$$
\begin{gathered}
-\frac{i}{\beta} \frac{1}{2 i} \oint_{c_{\tilde{p}}^{\prime}} d \tilde{p} \frac{(-1)^{\tilde{p}}}{\sin (\pi \tilde{p})} e^{i \frac{\tilde{p}}{\beta}\left(\tau_{2}-\tau_{1}\right)} e^{-i \frac{\pi}{2 \beta} \tilde{p}\left(\rho_{2}-\rho_{1}\right)} e^{-i \frac{\pi}{\beta} u_{0}[\tilde{p}]\left(\rho_{2}-\rho_{1}\right)} \frac{e^{-i \frac{3 \pi}{4 \beta}\left(\rho_{2}-\rho_{1}\right)}}{\sqrt{\rho_{1} \rho_{2}}} \times \\
\int_{0}^{\infty} d w \frac{e^{-\frac{\pi}{\beta} w\left(\rho_{2}-\rho_{1}\right)}}{\pi^{2}\left(u_{0}^{2}[\tilde{p}]-w^{2}\right)+4\left(l^{2}+l+1\right)-2 i \pi^{2} u_{0}[\tilde{p}] w}
\end{gathered}
$$

along the infinite contour $c_{\tilde{p}}^{\prime}$ of Fig.3. Replacing (19), (20) and (21) in the integral over $\tilde{p}$ in (40) taken along the large quadrant of $c_{\tilde{p}}^{\prime}$ and following the procedure relevant to (22) and (23) causes the stated integral to vanish at $|\tilde{p}| \rightarrow \infty$.

The integral over $\tilde{p}$ in (40), now taken along that segment of $c_{\tilde{p}}^{\prime}$ which lies on the imaginary axis, corresponds to $\theta_{\tilde{p}}=\frac{\pi}{2}$. In the context of (19), (20) and (21) that integral is

$$
\begin{gathered}
\tilde{I}_{c_{\tilde{p}}^{\prime}}^{\frac{\pi}{2}}=2 i \int_{\infty}^{\epsilon \rightarrow 0} d|\tilde{p}| \frac{1}{1-e^{-2 \pi|\tilde{p}|}} e^{i \frac{|\tilde{p}|}{\beta}\left(\xi_{2}-\xi_{1}\right)} e^{-i \frac{\pi}{2 \beta}|\tilde{p}|\left(\zeta_{2}-\zeta_{1}\right)} e^{-i \frac{\pi}{\beta} u_{0}[|\tilde{p}|]\left(\zeta_{2}-\zeta_{1}\right)} \frac{e^{-\frac{3 \pi}{4 \beta}\left(\zeta_{2}-\zeta_{1}\right)}}{\sqrt{\zeta_{1} \zeta_{2}}} \times \\
\int_{0}^{\infty} d w \frac{e^{i \frac{\pi}{\beta} w\left(\zeta_{2}-\zeta_{1}\right)}}{-\pi^{2}\left(u_{0}^{2}[|\tilde{p}|]+w^{2}\right)+4\left(l^{2}+l+1\right)+2 \pi^{2} u_{0}[|\tilde{p}|] w}
\end{gathered}
$$


In effect, the integral over $\tilde{p}$ in $(40)$ is equal to $\tilde{I}_{c_{\tilde{p}}^{\prime}}^{+}+\tilde{I}_{c_{\tilde{p}}^{\prime}}^{\frac{\pi}{2}}+\tilde{I}_{c_{\tilde{p}}^{\prime}}^{s c}$ - with the notation relevant to the first and the third term having the same significance as $I_{c_{\tilde{p}}^{\prime}}^{+}$and $I_{c_{\tilde{p}}^{\prime}}^{s c}$ respectively.

Again, the evaluation of the Cauchy principal value $\tilde{I}_{c_{\tilde{p}}^{\prime}}^{+}$necessitates the corresponding evaluation of the integral over $\tilde{p}$ in (40) along the infinite contour $\tilde{c}_{\tilde{p}}$ of Fig.4. Replacing (19), (20) and (21) in the integral over $\tilde{p}$ in (40) taken along the large quadrant of $\tilde{c}_{\tilde{p}}$ and following the procedure relevant to (26) and (27) causes the stated integral to vanish at $|\tilde{p}| \rightarrow \infty$.

The integral over $\tilde{p}$ in (40), now taken along that segment of $\tilde{c}_{\tilde{p}}$ which lies on the imaginary axis, corresponds to $\theta_{\tilde{p}}=-\frac{\pi}{2}$. Always in view of (19), (20) and (21) that integral is

$$
\begin{gathered}
\tilde{I}_{\tilde{c}_{\tilde{p}}^{-\frac{\pi}{2}}}=-2 i \int_{\infty}^{\epsilon \rightarrow 0} d|\tilde{p}| \frac{1}{1-e^{-2 \pi|\tilde{p}|} e^{i \frac{|\tilde{p}|}{\beta}\left(\xi_{2}-\xi_{1}\right)} e^{-i \frac{\pi}{2 \beta}|\tilde{p}|\left(\zeta_{2}-\zeta_{1}\right)} e^{-i \frac{\pi}{\beta} u_{0}[|\tilde{p}|]\left(\zeta_{2}-\zeta_{1}\right)} \frac{e^{\frac{3 \pi}{4 \beta}\left(\zeta_{2}-\zeta_{1}\right)}}{\sqrt{\zeta_{1} \zeta_{2}}} \times} \\
\int_{0}^{\infty} d w \frac{e^{-i \frac{\pi}{\beta} w\left(\zeta_{2}-\zeta_{1}\right)}}{-\pi^{2}\left(u_{0}^{2}[|\tilde{p}|]+w^{2}\right)+4\left(l^{2}+l+1\right)-2 \pi^{2} u_{0}[|\tilde{p}|] w}
\end{gathered}
$$

At once, Cauchy's theorem yields a vanishing result for the integral in (40) taken along $\tilde{c}_{\tilde{p}}$. Consequently, (42) implies

$$
\begin{gathered}
\tilde{I}_{c_{\tilde{p}}^{\prime}}^{+}=-2 i \int_{\epsilon \rightarrow 0}^{\infty} d|\tilde{p}| \frac{1}{1-e^{-2 \pi|\tilde{p}|}} e^{i \frac{|\tilde{p}|}{\beta}\left(\xi_{2}-\xi_{1}\right)} e^{-i \frac{\pi}{2 \beta}|\tilde{p}|\left(\zeta_{2}-\zeta_{1}\right)} e^{-i \frac{\pi}{\beta} u_{0}[|\tilde{p}|]\left(\zeta_{2}-\zeta_{1}\right)} \frac{e^{\frac{3 \pi}{4 \beta}\left(\zeta_{2}-\zeta_{1}\right)}}{\sqrt{\zeta_{1} \zeta_{2}}} \times \\
\int_{0}^{\infty} d w \frac{e^{-i \frac{\pi}{\beta} w\left(\zeta_{2}-\zeta_{1}\right)}}{-\pi^{2}\left(u_{0}^{2}[|\tilde{p}|]+w^{2}\right)+4\left(l^{2}+l+1\right)-2 \pi^{2} u_{0}[|\tilde{p}|] w}
\end{gathered}
$$

Finally, at $|\tilde{p}| \rightarrow 0$ the contribution $\tilde{I}_{c_{\tilde{p}}^{\prime}}^{s c}$ stemming from the integral along the stated small semi-circle in Fig.3 is

$$
\begin{gathered}
\tilde{I}_{c_{\tilde{p}}}^{s c}=\frac{i}{\pi} \frac{1}{\sqrt{\zeta_{1} \zeta_{2}}} \int_{\frac{\pi}{2}}^{\frac{3 \pi}{2}} d \theta e^{i \theta} e^{-i \frac{\pi}{\beta}\left[u_{0}[0]+\frac{3}{4} e^{-i \theta}\right]\left(\zeta_{2}-\zeta_{1}\right)} \times \\
\int_{0}^{\infty} d w \frac{e^{-\frac{\pi}{\beta} w e^{-i \theta}\left(\zeta_{2}-\zeta_{1}\right)}}{\pi^{2}\left(u_{0}^{2}[0] e^{2 i \theta}-w^{2}\right)+4\left(l^{2}+l+1\right)-2 i \pi^{2} u_{0}[0] e^{i \theta} w}
\end{gathered}
$$

As stated, the integral over $\tilde{p}$ in (40) taken along the infinite contour $c_{\tilde{p}}^{\prime}$ of Fig.3 is equal to $\tilde{I}_{c_{\tilde{p}}^{\prime}}^{+}+\tilde{I}_{c_{\tilde{p}}^{\prime}}^{\frac{\pi}{2}}+\tilde{I}_{c_{\tilde{p}}^{\prime}}^{s c}$. Hence, as a result of $(43),(41)$ and (44) it is

$$
\begin{gathered}
-\frac{1}{2 \beta} \oint_{c_{\tilde{p}}^{\prime}} d \tilde{p} \frac{(-1)^{\tilde{p}}}{\sin (\pi \tilde{p})} e^{i \frac{\tilde{p}}{\beta}\left(\tau_{2}-\tau_{1}\right)} e^{-i \frac{\pi}{2 \beta} \tilde{p}\left(\rho_{2}-\rho_{1}\right)} e^{-i \frac{\pi}{\beta} u_{0}[\tilde{p}]\left(\rho_{2}-\rho_{1}\right)} \frac{e^{-i \frac{3 \pi}{4 \beta}\left(\rho_{2}-\rho_{1}\right)}}{\sqrt{\rho_{1} \rho_{2}}} \times \\
\int_{0}^{\infty} d w \frac{e^{-\frac{\pi}{\beta} w\left(\rho_{2}-\rho_{1}\right)}}{\pi^{2}\left(u_{0}^{2}[\tilde{p}]-w^{2}\right)+4\left(l^{2}+l+1\right)-2 i \pi^{2} u_{0}[\tilde{p}] w}=
\end{gathered}
$$




$$
\begin{gathered}
\frac{i}{\beta} \frac{e^{\frac{3 \pi}{4 \beta}\left(\zeta_{2}-\zeta_{1}\right)}}{\sqrt{\zeta_{1} \zeta_{2}}} \int_{\epsilon \rightarrow 0}^{\infty} d|\tilde{p}| \frac{1}{1-e^{-2 \pi|\tilde{p}|}} e^{i \frac{\mid \tilde{p}}{\beta}\left(\xi_{2}-\xi_{1}\right)} e^{-i \frac{\pi}{\beta}\left[\frac{|\tilde{p}|}{2}+u_{0}[|\tilde{p}|]\right]\left(\zeta_{2}-\zeta_{1}\right)} \times \\
\int_{0}^{\infty} d w \frac{e^{-i \frac{\pi}{\beta} w\left(\zeta_{2}-\zeta_{1}\right)}}{-\pi^{2}\left(u_{0}^{2}[|\tilde{p}|]+w^{2}\right)+4\left(l^{2}+l+1\right)-2 \pi^{2} u_{0}[|\tilde{p}|] w} \\
+\frac{i}{\beta} \frac{e^{-\frac{3 \pi}{4 \beta}\left(\zeta_{2}-\zeta_{1}\right)}}{\sqrt{\zeta_{1} \zeta_{2}}} \int_{\epsilon \rightarrow 0}^{\infty} d|\tilde{p}| \frac{1}{1-e^{-2 \pi|\tilde{p}|} e^{i \frac{\tilde{p}}{\beta}\left(\zeta_{2}-\xi_{1}\right)} e^{-i \frac{\pi}{\beta}\left[\frac{|\tilde{p}|}{2}+u_{0}[|\tilde{p}|]\right]\left(\zeta_{2}-\zeta_{1}\right)} \times} \\
\int_{0}^{\infty} d w \frac{e^{i \frac{\pi}{\beta} w\left(\zeta_{2}-\zeta_{1}\right)}}{-\pi^{2}\left(u_{0}^{2}[|\tilde{p}|]+w^{2}\right)+4\left(l^{2}+l+1\right)+2 \pi^{2} u_{0}[|\tilde{p}|] w} \\
-\frac{i}{\beta} \frac{1}{2 \pi} \frac{e^{-i \frac{\pi}{\beta} u_{0}[0]\left(\zeta_{2}-\zeta_{1}\right)}}{\sqrt{\zeta_{1} \zeta_{2}}} \int_{\frac{\pi}{2}}^{\frac{3 \pi}{2}} d \theta e^{i \theta} e^{-i \frac{3 \pi}{4 \beta} e^{-i \theta}\left(\zeta_{2}-\zeta_{1}\right)} \times \\
\int_{0}^{\infty} d w \frac{e^{-\frac{\pi}{\beta} w e^{-i \theta}\left(\zeta_{2}-\zeta_{1}\right)}}{\pi^{2}\left(u_{0}^{2}[0] e^{2 i \theta}-w^{2}\right)+4\left(l^{2}+l+1\right)-2 i \pi^{2} u_{0}[0] e^{i \theta} w}
\end{gathered}
$$

This is the analytical extension of (12). The analysis as to the physical significance of (33) applies, of course, identically also to (45). It should be remarked, in addition, that the last term in (33) and the corresponding last term in (45) appear to be somewhat peculiar as they do not feature any time dependence. However, the presence of these two terms in the final result of the analytical extension should not be surprising. By the analytical procedure in the $\tilde{p}$-complex plane it is clear that they correspond to the $p=0$ terms in (11) and (12) respectively. What may come as a surprise, nevertheless, is that these two "innocuous-looking" terms will prove to be the very cause of the radiation of particles by the Schwarzschild black hole, as will be seen in section VI.

In the interior of the Schwarzschild black hole the additive result of (33) and (45) is, in $1+1$ dimensions, the physically valid analytical extension of the singular part $D_{a s}\left(x_{2}-x_{1}\right)$ of the Green function in (6).

\section{The Analytical Extension - Boundary Part}

The boundary part $D_{b}\left(x_{2}-x_{1}\right)$ of the Green function in (6) - that is, the part which remains finite at $x_{2} \rightarrow x_{1}$ and enforces the boundary condition [4]

is

$$
D\left(x_{2}-x_{1}\right)_{\mid \rho_{2}=\beta \bigvee \rho_{1}=\beta}=0
$$

$$
D_{b}\left(x_{2}-x_{1}\right)=-\frac{2}{\beta^{\frac{3}{2}}} \frac{1}{\sqrt{\rho_{1}}} \times
$$

$$
\sum_{l=0}^{\infty} \sum_{m=-l}^{l} \sum_{p=0}^{\infty} \int_{u_{0}^{\prime}[p]}^{\infty} d u \frac{\cos \left[\frac{\pi}{4 \beta}(4 u+2 p+3)\left(\beta-\rho_{1}\right)\right]}{\pi^{2} u^{2}+4\left(l^{2}+l+1\right)} \frac{J_{p}\left(\frac{2 i}{\beta} \sqrt{l^{2}+l+1} \rho_{2}\right)}{J_{p}\left(2 i \sqrt{l^{2}+l+1}\right)} e^{i \frac{p}{\beta}\left(\tau_{2}-\tau_{1}\right)} Y_{l m}\left(\theta_{2}, \phi_{2}\right) Y_{l m}^{*}\left(\theta_{1}, \phi_{1}\right) ;
$$

the radial-temporal sector of which is the sum of 


$$
-\frac{1}{\beta^{\frac{3}{2}}} \frac{e^{i \frac{3 \pi}{4 \beta}\left(\beta-\rho_{1}\right)}}{\sqrt{\rho_{1}}} \sum_{p=0}^{\infty} e^{i \frac{p}{\beta}\left(\tau_{2}-\tau_{1}\right)} e^{i \frac{\pi}{2 \beta} p\left(\beta-\rho_{1}\right)} \frac{J_{p}\left(\frac{2 i}{\beta} \sqrt{l^{2}+l+1} \rho_{2}\right)}{J_{p}\left(2 i \sqrt{l^{2}+l+1}\right)} \int_{u_{0}^{\prime}[p]}^{\infty} d u \frac{e^{i \frac{\pi}{\beta} u\left(\beta-\rho_{1}\right)}}{\pi^{2} u^{2}+4\left(l^{2}+l+1\right)}
$$

and

$$
-\frac{1}{\beta^{\frac{3}{2}}} \frac{e^{-i \frac{3 \pi}{4 \beta}\left(\beta-\rho_{1}\right)}}{\sqrt{\rho_{1}}} \sum_{p=0}^{\infty} e^{i \frac{p}{\beta}\left(\tau_{2}-\tau_{1}\right)} e^{-i \frac{\pi}{2 \beta} p\left(\beta-\rho_{1}\right)} \frac{J_{p}\left(\frac{2 i}{\beta} \sqrt{l^{2}+l+1} \rho_{2}\right)}{J_{p}\left(2 i \sqrt{l^{2}+l+1}\right)} \int_{u_{0}^{\prime}[p]}^{\infty} d u \frac{e^{-i \frac{\pi}{\beta} u\left(\beta-\rho_{1}\right)}}{\pi^{2} u^{2}+4\left(l^{2}+l+1\right)}
$$

The expressions in (47) and (48) are valid in the exterior region of the Schwarzschild black-hole space-time and, as before, the replacement of $r \geq 2 M$ by $0 \leq r<2 M$ causes either one of them to diverge sharply for arbitrary space-time separations. It would appear, for that matter, that the analytical procedure which established a convergent expression for the singular part of the propagator in the interior region can also establish a convergent expression for the propagator's boundary part. In the present case, however, there is an additional issue which stems from the fact that for fixed $z \in C$ the Bessel functions of the first and second kind, $J_{\nu}(z)$ and $I_{\nu}(z)$ respectively, have an essential singularity at $|\nu| \rightarrow \infty$ [6]. In effect, in order to implement the stated analytical procedure it must first be established that, upon replacing (16) with $\rho_{2}=\beta$ in (47), the meromorphic 5 function which constitutes the integrand in

$$
\begin{gathered}
-\frac{i}{\beta^{\frac{3}{2}}} \frac{1}{2 i} \oint d \tilde{p} \frac{(-1)^{\tilde{p}}}{\sin (\pi \tilde{p})} e^{i \frac{\tilde{p}}{\beta}\left(\tau_{2}-\tau_{1}\right)} e^{i \frac{\pi}{2 \beta} \tilde{p}\left(\beta-\rho_{1}\right)} e^{i \frac{\pi}{\beta} u_{0}^{\prime}[\tilde{p}]\left(\beta-\rho_{1}\right)} \frac{e^{i \frac{3 \pi}{4 \beta}\left(\beta-\rho_{1}\right)}}{\sqrt{\rho_{1}}} \frac{J_{\tilde{p}\left(\frac{2 i}{\beta} \sqrt{l^{2}+l+1} \rho_{2}\right)}}{J_{\tilde{p}}\left(2 i \sqrt{l^{2}+l+1}\right)} \times \\
\quad \int_{0}^{\infty} d w \frac{e^{-\frac{\pi}{\beta} w\left(\beta-\rho_{1}\right)}}{\pi^{2}\left(u_{0}^{\prime 2}[\tilde{p}]-w^{2}\right)+4\left(l^{2}+l+1\right)+2 i \pi^{2} u_{0}^{\prime}[\tilde{p}] w}
\end{gathered}
$$

taken along the infinite contours $c_{\tilde{p}}^{\prime}$ and $\tilde{c}_{\tilde{p}}$ in Fig.3 and Fig.4 respectively contains no essential singularities also at $|\tilde{p}| \rightarrow \infty$. In order to explore the behaviour of the integrand at $|\tilde{p}| \rightarrow \infty$ use must be made of the representation

$$
J_{\nu}(z)=\left(\frac{z}{2}\right)^{\nu} \sum_{s=0}^{\infty} \frac{(-1)^{s}\left(\frac{z^{2}}{4}\right)^{s}}{s ! \Gamma(\nu+s+1)} ; z \in C, \nu \in C
$$

which implies that, for $|\tilde{p}|>>1$, the ratio of the two Bessel functions in (49) behaves as

$$
\frac{J_{\tilde{p}}\left(\frac{2 i}{\beta} \sqrt{l^{2}+l+1} \rho_{2}\right)}{J_{\tilde{p}}\left(2 i \sqrt{l^{2}+l+1}\right)} \approx e^{\tilde{p} \ln \frac{\rho_{2}}{\beta}}\left[1+O\left(\frac{1}{\tilde{p}}\right)\right]
$$

\footnotetext{
${ }^{5}$ It is meromorphic in the sense that all branch cuts exist exclusively in the complexified coordinate domain. There are no cuts in the $\tilde{p}$-plane. This point is further elaborated below.
} 
As was the case with the singular part it can be seen again that (47) admits several analytical extensions depending on the manner in which $(-1)^{\tilde{p}}$ is expressed in (49). The condition which this time selects a unique, physically valid analytical extension is, of course, that the boundary part - and consequently the analytical extension of (47) and (48) independently - be finite for all values of $\xi_{2}-\xi_{1}$ and $\zeta_{2}-\zeta_{1}$. As a consequence, any extension which does not meet that requirement must be ruled out. At once, consistency with this requirement imposes the additional demand that the analytical extension of (47) and, independently, that of (48) yield a finite result for all values of $\xi_{2,1}$ and $\zeta_{2,1}$ at each stage of the calculation.

As a consequence of this demand it is $(-1)^{\tilde{p}}=\left(e^{i \pi}\right)^{\tilde{p}}$, if the integral in (49) is taken along $c_{\tilde{p}}^{\prime}$. In that case the product between $(51)$ and the asymptotic expression of $\frac{(-1)^{\tilde{p}}}{\sin (\pi \tilde{p})}$ is

$$
2 e^{|\tilde{p}|\left(\cos \theta \ln \frac{\zeta_{2}}{\beta}+(\theta-2 \pi) \sin \theta\right)} e^{i|\tilde{p}|\left(\sin \theta \ln \frac{\zeta_{2}}{\beta}-(\theta-2 \pi) \cos \theta\right)}\left[1+O\left(\frac{1}{|\tilde{p}|}\right)\right]
$$

In view of the fact that $\zeta<\beta$ and that, in this case, it is $0 \leq \theta \leq \frac{\pi}{2}$ this product causes the integral in (49) to vanish exponentially as $|\tilde{p}| \rightarrow \infty$.

Likewise, if the integrand in (49) is taken along $\tilde{c}_{\tilde{p}}$ the demand that the analytical extension of (47) yield a finite result for all values of $\xi_{2,1}$ and $\zeta_{2,1}$ at each stage of the calculation implies that $(-1)^{\tilde{p}}=\left(e^{-i \pi}\right)^{\tilde{p}}$. In that case the product between $(51)$ and the asymptotic expression of $\frac{(-1)^{\tilde{p}}}{\sin (\pi \tilde{p})}$ is

$$
2 e^{|\tilde{p}|\left(\cos \theta \ln \frac{\zeta_{2}}{\beta}+(\theta+2 \pi) \sin \theta\right)} e^{i|\tilde{p}|\left(\sin \theta \ln \frac{\zeta_{2}}{\beta}-(\theta+2 \pi) \cos \theta\right)}\left[1+O\left(\frac{1}{|\tilde{p}|}\right)\right]
$$

and causes the integral in (49) to vanish as $|\tilde{p}| \rightarrow \infty$ for all values of $\theta \in\left(0,-\frac{\pi}{2}\right]$.

In effect, the analytical procedure which yielded the extension of $D_{a s}\left(x_{2}-x_{1}\right)$ in (33) and (45) can also be applied to (49) to the same purpose. Before advancing that procedure, however, it is important to note that - as was also the case in (18) and (40) for each value of $\tilde{p} \in C$ the integrand of the contour integral in (49) is a multiple-valued function of $\tau_{2}-\tau_{1}$ although the contour integral itself is a single-valued function of that temporal difference. Unlike (18) and (40), however, the integrand featured in (49) is - for each value of $\tilde{p}$ - also a multiple-valued function of $\rho_{2}$. This is a direct consequence of the fact that the Bessel functions of the first and second kind, $J_{\nu}(z)$ and $I_{\nu}(z)$ respectively, are multiple-valued functions in the $z$-complex plane unless $\nu \in Z$ [6] 6. Despite that fact it follows again from the character of (49) as an integral representation of (47) that the contour integral in (49) is, itself, also a single-valued function of $\rho_{2}$. At once, the contour integral in (49) is - despite appearances - a single-valued function of $\rho_{1}$. This follows again from the implicit dependence of $\rho_{1}$ on $\tilde{p}$ through (21) and the fact that (49) is an integral representation of (47). Consequently, the physical demand that the propagator be regular for all values of space-time coordinates in its domain is satisfied in the interior of the Schwarzschild black hole.

\footnotetext{
${ }^{6}$ This situation is reminiscent of that in [7] and [2] in which the unknown functions of the radial coordinate have been shown to feature branch cuts in the corresponding radial variable.
} 
The ensuing analysis follows the same pattern as that which was applied to the singular part $D_{a s}\left(x_{2}-x_{1}\right)$. In view of the stated fact that the contour integral in (49) vanishes along the infinite quadrant of $c_{\tilde{p}}^{\prime}$ it follows that - in the context of (19), (20) and (21) the contribution which that contour integral receives along that segment of $c_{\tilde{p}}^{\prime}$ which lies on the imaginary axis is

$$
\begin{gathered}
\mathbf{I}_{c_{\tilde{p}}^{\prime}}^{\frac{\pi}{2}}=\int_{\infty}^{\epsilon \rightarrow 0} d|\tilde{p}| \frac{2}{e^{2 \pi|\tilde{p}|}-1} e^{i \frac{|\tilde{p}|}{\beta}\left(\xi_{2}-\xi_{1}\right)} e^{i \frac{\pi}{\beta}\left[\frac{|\tilde{p}|}{2}+u_{0}^{\prime}[|\tilde{p}|]\right]\left(\beta-\zeta_{1}\right)} \frac{e^{\frac{3 \pi}{4 \beta}\left(\beta-\zeta_{1}\right)}}{\frac{\sqrt{2}}{2}(1-i) \sqrt{\zeta_{1}}} \times \\
\frac{J_{i|\tilde{p}|}\left(-\frac{2}{\beta} \sqrt{l^{2}+l+1} \zeta_{2}\right)}{J_{i|\tilde{p}|}\left(-2 i \sqrt{l^{2}+l+1}\right)} \int_{0}^{\infty} d w \frac{e^{i \frac{\pi}{\beta} w\left(\beta-\zeta_{1}\right)}}{-\pi^{2}\left(u_{0}^{\prime 2}[|\tilde{p}|]+w^{2}\right)+4\left(l^{2}+l+1\right)-2 \pi^{2} u_{0}^{\prime}[|\tilde{p}|] w}
\end{gathered}
$$

In arriving at (54) the identities

$$
\begin{aligned}
& J_{\nu}(z)=\frac{z^{\nu}}{(i z)^{\nu}} I_{\nu}(i z) \\
& J_{\nu}(i z)=\frac{(i z)^{\nu}}{z^{\nu}} I_{\nu}(z) \\
& I_{\nu}(z)=\frac{z^{\nu}}{(i z)^{\nu}} J_{\nu}(i z) \\
& I_{\nu}(i z)=\frac{(i z)^{\nu}}{z^{\nu}} J_{\nu}(z)
\end{aligned}
$$

have also been used. It should be remarked that

$$
\frac{J_{i|\tilde{p}|}\left(-\frac{2}{\beta} \sqrt{l^{2}+l+1} \zeta_{2}\right)}{J_{i|\tilde{p}|}\left(-2 i \sqrt{l^{2}+l+1}\right)}=\frac{J_{i|\tilde{p}|}\left(\frac{2}{\beta} \sqrt{l^{2}+l+1} \zeta_{2}\right)}{J_{i|\tilde{p}|}\left(2 i \sqrt{l^{2}+l+1}\right)}
$$

the right side of which identity could have been directly obtained for $\theta=\frac{\pi}{2}$ in (49) that is, without use of the identities in (55) - (58). The expression in (54) must be understood as equivalent to that stemming from the right side of (59) as well as to all other expressions allowed by the identities in (55) - (58).

An immediate consequence of the expression in (52) is

$$
\frac{J_{i|\tilde{p}|}\left(-\frac{2}{\beta} \sqrt{l^{2}+l+1} \zeta_{2}\right)}{J_{i|\tilde{p}|}\left(-2 i \sqrt{l^{2}+l+1}\right)} \approx e^{\frac{\pi}{2}|\tilde{p}|} e^{i|\tilde{p}| \ln \frac{\zeta_{2}}{\beta}}\left[1+0\left(\frac{1}{|\tilde{p}|}\right)\right]
$$

as a result of which the expression in (54) vanishes at $|\tilde{p}| \rightarrow \infty$.

As was the case with the contour integrals over $\tilde{p}$ in (18) and (40) the integral over $\tilde{p}$ in (49) taken along the infinite contour $c_{\tilde{p}}^{\prime}$ is equal to $\mathbf{I}_{c_{\tilde{p}}^{\prime}}^{+}+\mathbf{I}_{c_{\tilde{p}}^{\prime}}^{\frac{\pi}{2}}+\mathbf{I}_{c_{\tilde{p}}^{\prime}}^{S C}$ with $\mathbf{I}_{c_{\tilde{p}}^{\prime}}^{+}$being the contribution which that contour integral receives along $c_{\tilde{p}}^{+}$and with $\mathbf{I}_{c_{\tilde{p}}^{\prime}}^{S C}$ being the contribution along the small semi-circle centred at zero and extending from $\theta_{\tilde{p}}=\frac{\pi}{2}$ to 
$\theta_{\tilde{p}}=\frac{3 \pi}{2}$ in Fig.3. In view of the stated fact that the contour integral in (49) vanishes along the infinite quadrant of $\tilde{c}_{\tilde{p}}$ in Fig.4 the Cauchy principal value $\mathbf{I}_{c_{\tilde{p}}^{\prime}}^{+}$can - as before be evaluated through use of Cauchy's theorem applied to the integral in (49) taken along $\tilde{c}_{\tilde{p}}$.

In the context of (55) to (58) the contribution which the contour integral in (49) receives along that segment of $\tilde{c}_{\tilde{p}}$ which lies on the imaginary axis is

$$
\begin{aligned}
& \mathbf{I}_{\tilde{c}_{\tilde{p}}}^{-\frac{\pi}{2}}=\int_{\infty}^{\epsilon \rightarrow 0} d|\tilde{p}| \frac{2}{e^{2 \pi|\tilde{p}|}-1} e^{i \frac{|\tilde{p}|}{\beta}\left(\xi_{2}-\xi_{1}\right)} e^{i \frac{\pi}{2 \beta}|\tilde{p}|\left(\beta-\zeta_{1}\right)} e^{i \frac{\pi}{\beta} u_{0}^{\prime}[\tilde{p} \mid]\left(\beta-\zeta_{1}\right)} \frac{e^{-\frac{3 \pi}{4 \beta}\left(\beta-\zeta_{1}\right)}}{\frac{\sqrt{2}}{2}(1+i) \sqrt{\zeta_{1}}} \times \\
& \frac{J_{-i|\tilde{p}|}\left(\frac{2}{\beta} \sqrt{l^{2}+l+1} \zeta_{2}\right)}{J_{-i|\tilde{p}|}\left(-2 i \sqrt{l^{2}+l+1}\right)} \int_{0}^{\infty} d w \frac{e^{-i \frac{\pi}{\beta} w\left(\beta-\zeta_{1}\right)}}{-\pi^{2}\left(u_{0}^{\prime 2}[|\tilde{p}|]+w^{2}\right)+4\left(l^{2}+l+1\right)+2 \pi^{2} u_{0}^{\prime}[|\tilde{p}|] w}
\end{aligned}
$$

Parenthetically, it shall be remarked again that - pursuant to (59) - one of the several expressions equivalent to (61) can be obtained from the identity

$$
\frac{J_{-i|\tilde{p}|}\left(\frac{2}{\beta} \sqrt{l^{2}+l+1} \zeta_{2}\right)}{J_{-i|\tilde{p}|}\left(-2 i \sqrt{l^{2}+l+1}\right)}=\frac{J_{-i|\tilde{p}|}\left(-\frac{2}{\beta} \sqrt{l^{2}+l+1} \zeta_{2}\right)}{J_{-i|\tilde{p}|}\left(2 i \sqrt{l^{2}+l+1}\right)}
$$

An immediate consequence of the expression in (53) is

$$
\frac{J_{-i|\tilde{p}|}\left(\frac{2}{\beta} \sqrt{l^{2}+l+1} \zeta_{2}\right)}{J_{-i|\tilde{p}|}\left(-2 i \sqrt{l^{2}+l+1}\right)} \approx e^{\frac{\pi}{2}|\tilde{p}|} e^{-i|\tilde{p}| l n \frac{\zeta_{2}}{\beta}}\left[1+0\left(\frac{1}{|\tilde{p}|}\right)\right]
$$

as a result of which the expression in (61) vanishes at $|\tilde{p}| \rightarrow \infty$.

Since the function which in (49) is integrated over $\tilde{p}$ is regular throughout the region bounded by the infinite contour $\tilde{c}_{\tilde{p}}$ it follows that

$$
\begin{gathered}
\mathbf{I}_{c_{\tilde{p}}^{\prime}}^{+}=\int_{\epsilon \rightarrow 0}^{\infty} d|\tilde{p}| \frac{2}{e^{2 \pi|\tilde{p}|}-1} e^{i \frac{\tilde{p} \mid}{\beta}\left(\xi_{2}-\xi_{1}\right)} e^{i \frac{\pi}{\beta}\left[\frac{|\tilde{p}|}{2}+u_{0}^{\prime}[|\tilde{p}|]\right]\left(\beta-\zeta_{1}\right)} \frac{e^{-\frac{3 \pi}{4 \beta}\left(\beta-\zeta_{1}\right)}}{\frac{\sqrt{2}}{2}(1+i) \sqrt{\zeta_{1}}} \times \\
\frac{J_{-i|\tilde{p}|}\left(\frac{2}{\beta} \sqrt{l^{2}+l+1} \zeta_{2}\right)}{J_{-i|\tilde{p}|}\left(-2 i \sqrt{l^{2}+l+1}\right)} \int_{0}^{\infty} d w \frac{e^{-i \frac{\pi}{\beta} w\left(\beta-\zeta_{1}\right)}}{-\pi^{2}\left(u_{0}^{\prime 2}[|\tilde{p}|]+w^{2}\right)+4\left(l^{2}+l+1\right)+2 \pi^{2} u_{0}^{\prime}[|\tilde{p}|] w}
\end{gathered}
$$

Finally, it can readily be verified that at $|\tilde{p}| \rightarrow 0$ the contribution stemming from the integral along the stated small semi-circle in Fig.3 is

$$
\begin{aligned}
\mathbf{I}_{c_{\tilde{p}}^{\prime}}^{S C}= & \frac{i}{\pi} \frac{1}{\sqrt{\zeta_{1}}} \int_{\frac{\pi}{2}}^{\frac{3 \pi}{2}} d \theta e^{i \frac{\theta}{2}} e^{i \frac{\pi}{\beta}\left[u_{0}^{\prime}[0]+\frac{3}{4} e^{-i \theta}\right]\left(\beta-\zeta_{1}\right)} \frac{I_{0}\left(\frac{2}{\beta} \sqrt{l^{2}+l+1} e^{-i \theta} \zeta_{2}\right)}{I_{0}\left(2 \sqrt{l^{2}+l+1}\right)} \times \\
& \int_{0}^{\infty} d w \frac{e^{-\frac{\pi}{\beta} w e^{-i \theta}\left(\beta-\zeta_{1}\right)}}{\pi^{2}\left(u_{0}^{\prime 2}[0] e^{2 i \theta}-w^{2}\right)+4\left(l^{2}+l+1\right)+2 i \pi^{2} u_{0}^{\prime}[0] e^{i \theta} w}
\end{aligned}
$$


As stated, the contour integral over $\tilde{p}$ in (49) taken along the infinite contour $c_{\tilde{p}}^{\prime}$ in Fig.3 is equal to $\mathbf{I}_{c_{\tilde{p}}^{\prime}}^{\frac{\pi}{2}}+\mathbf{I}_{c_{\tilde{p}}^{\prime}}^{+}+\mathbf{I}_{c_{\tilde{p}}^{\prime}}^{S C}$. Hence, as a result of (54), (64) and (65) it is

$$
\begin{aligned}
& -\frac{1}{2 \beta^{\frac{3}{2}}} \oint_{c_{\tilde{p}}^{\prime}} d \tilde{p} \frac{(-1)^{\tilde{p}}}{\sin (\pi \tilde{p})} e^{i \frac{\tilde{p}}{\beta}\left(\tau_{2}-\tau_{1}\right)} e^{i \frac{\pi}{2 \beta} \tilde{p}\left(\beta-\rho_{1}\right)} e^{i \frac{\pi}{\beta} u_{0}^{\prime}[\tilde{p}]\left(\beta-\rho_{1}\right)} \frac{e^{i \frac{3 \pi}{4 \beta}\left(\beta-\rho_{1}\right)}}{\sqrt{\rho_{1}}} \frac{J_{\tilde{p}}\left(\frac{2 i}{\beta} \sqrt{l^{2}+l+1} \rho_{2}\right)}{J_{\tilde{p}}\left(2 i \sqrt{l^{2}+l+1}\right)} \times \\
& \int_{0}^{\infty} d w \frac{e^{-\frac{\pi}{\beta} w\left(\beta-\rho_{1}\right)}}{\pi^{2}\left(u_{0}^{\prime 2}[\tilde{p}]-w^{2}\right)+4\left(l^{2}+l+1\right)+2 i \pi^{2} u_{0}^{\prime}[\tilde{p}] w}= \\
& +\frac{\sqrt{2}}{\beta^{\frac{3}{2}}} \frac{e^{\frac{3 \pi}{4 \beta}\left(\beta-\zeta_{1}\right)}}{(1-i) \sqrt{\zeta_{1}}} \int_{\epsilon \rightarrow 0}^{\infty} d|\tilde{p}| \frac{1}{e^{2 \pi|\tilde{p}|}-1} e^{i \frac{i \tilde{p} \mid}{\beta}\left(\xi_{2}-\xi_{1}\right)} e^{i \frac{\pi}{\beta}\left[\frac{|\tilde{p}|}{2}+u_{0}^{\prime}[|\tilde{p}|]\right]\left(\beta-\zeta_{1}\right)} \times \\
& \frac{J_{i|\tilde{p}|}\left(-\frac{2}{\beta} \sqrt{l^{2}+l+1} \zeta_{2}\right)}{J_{i|\tilde{p}|}\left(-2 i \sqrt{l^{2}+l+1}\right)} \int_{0}^{\infty} d w \frac{e^{i \frac{\pi}{\beta} w\left(\beta-\zeta_{1}\right)}}{-\pi^{2}\left(u_{0}^{2}[|\tilde{p}|]+w^{2}\right)+4\left(l^{2}+l+1\right)-2 \pi^{2} u_{0}^{\prime}[|\tilde{p}|] w} \\
& -\frac{\sqrt{2}}{\beta^{\frac{3}{2}}} \frac{e^{-\frac{3 \pi}{4 \beta}\left(\beta-\zeta_{1}\right)}}{(1+i) \sqrt{\zeta_{1}}} \int_{\epsilon \rightarrow 0}^{\infty} d|\tilde{p}| \frac{1}{e^{2 \pi|\tilde{p}|}-1} e^{i \frac{|\tilde{p}|}{\beta}\left(\xi_{2}-\xi_{1}\right)} e^{i \frac{\pi}{\beta}\left[\frac{|\tilde{p}|}{2}+u_{0}^{\prime}[|\tilde{p}|]\right]\left(\beta-\zeta_{1}\right)} \times \\
& \frac{J_{-i|\tilde{p}|}\left(\frac{2}{\beta} \sqrt{l^{2}+l+1} \zeta_{2}\right)}{J_{-i|\tilde{p}|}\left(-2 i \sqrt{l^{2}+l+1}\right)} \int_{0}^{\infty} d w \frac{e^{-i \frac{\pi}{\beta} w\left(\beta-\zeta_{1}\right)}}{-\pi^{2}\left(u_{0}^{\prime 2}[|\tilde{p}|]+w^{2}\right)+4\left(l^{2}+l+1\right)+2 \pi^{2} u_{0}^{\prime}[|\tilde{p}|] w} \\
& -\frac{i}{\beta^{\frac{3}{2}}} \frac{1}{2 \pi} \frac{e^{i \frac{\pi}{\beta} u_{0}^{\prime}[0]\left(\beta-\zeta_{1}\right)}}{\sqrt{\zeta_{1}}} \int_{\frac{\pi}{2}}^{\frac{3 \pi}{2}} d \theta e^{i \frac{\theta}{2}} e^{i \frac{3 \pi}{4 \beta} e^{-i \theta}\left(\beta-\zeta_{1}\right)} \frac{I_{0}\left(\frac{2}{\beta} \sqrt{l^{2}+l+1} e^{-i \theta} \zeta_{2}\right)}{I_{0}\left(2 \sqrt{l^{2}+l+1}\right)} \times \\
& \int_{0}^{\infty} d w \frac{e^{-\frac{\pi}{\beta} w e^{-i \theta}\left(\beta-\zeta_{1}\right)}}{\pi^{2}\left(u_{0}^{\prime 2}[0] e^{2 i \theta}-w^{2}\right)+4\left(l^{2}+l+1\right)+2 i \pi^{2} u_{0}^{\prime}[0] e^{i \theta} w}
\end{aligned}
$$

This is the analytical extension of (47). It is a consequence of the character of the integrand in (49) as a meromorphic function in the $\tilde{p}$-plane containing no essential singularities at infinity for $\theta_{\tilde{p}} \in\left[-\frac{\pi}{2}, \frac{\pi}{2}\right]$. As was the case in (33) and (45) each of the first two terms on the right side of (66) is a multiple-valued function of $\xi_{2}-\xi_{1}$. However, as already stated, the sum-total of the three terms on the right side of (66) - being an integral representation of (47) - is always a single-valued function of $\xi_{2}-\xi_{1}$ and of the radial coordinates $\rho_{2}$ and $\rho_{1}$.

The analytical extension of (48) follows the same pattern as that of (47). Replacing (38) with $\rho_{2}=\beta$ in (48) and converting the infinite series over $p$ to a contour integral over the contour $c_{\tilde{p}}^{\prime}$ yields

$$
\begin{gathered}
\frac{1}{2 \beta^{\frac{3}{2}}} \oint_{c_{\tilde{p}}^{\prime}} d \tilde{p} \frac{(-1)^{\tilde{p}}}{\sin (\pi \tilde{p})} e^{i \frac{\tilde{p}}{\beta}\left(\tau_{2}-\tau_{1}\right)} e^{-i \frac{\pi}{2 \beta} \tilde{p}\left(\beta-\rho_{1}\right)} e^{-i \frac{\pi}{\beta} u_{0}^{\prime}[\tilde{p}]\left(\beta-\rho_{1}\right)} \frac{e^{-i \frac{3 \pi}{4 \beta}\left(\beta-\rho_{1}\right)}}{\sqrt{\rho_{1}}} \frac{J_{\tilde{p}}\left(\frac{2 i}{\beta} \sqrt{l^{2}+l+1} \rho_{2}\right)}{J_{\tilde{p}}\left(2 i \sqrt{l^{2}+l+1}\right)} \times \\
\int_{0}^{\infty} d w \frac{e^{-\frac{\pi}{\beta} w\left(\beta-\rho_{1}\right)}}{\pi^{2}\left(u_{0}^{\prime 2}[\tilde{p}]-w^{2}\right)+4\left(l^{2}+l+1\right)-2 i \pi^{2} u_{0}^{\prime}[\tilde{p}] w}=
\end{gathered}
$$




$$
\begin{aligned}
& -\frac{\sqrt{2}}{\beta^{\frac{3}{2}}} \frac{e^{-\frac{3 \pi}{4 \beta}\left(\beta-\zeta_{1}\right)}}{(1-i) \sqrt{\zeta_{1}}} \int_{\epsilon \rightarrow 0}^{\infty} d|\tilde{p}| \frac{1}{e^{2 \pi|\tilde{p}|}-1} e^{i \frac{|\tilde{p}|}{\beta}\left(\xi_{2}-\xi_{1}\right)} e^{-i \frac{\pi}{\beta}\left[\frac{|\tilde{p}|}{2}+u_{0}^{\prime}[\tilde{p} \mid]\right]\left(\beta-\zeta_{1}\right)} \times \\
& \frac{J_{i|\tilde{p}|}\left(-\frac{2}{\beta} \sqrt{l^{2}+l+1} \zeta_{2}\right)}{J_{i|\tilde{p}|}\left(-2 i \sqrt{l^{2}+l+1}\right)} \int_{0}^{\infty} d w \frac{e^{i \frac{\pi}{\beta} w\left(\beta-\zeta_{1}\right)}}{-\pi^{2}\left(u_{0}^{\prime 2}[|\tilde{p}|]+w^{2}\right)+4\left(l^{2}+l+1\right)+2 \pi^{2} u_{0}^{\prime}[|\tilde{p}|] w} \\
& +\frac{\sqrt{2}}{\beta^{\frac{3}{2}}} \frac{e^{\frac{3 \pi}{4 \beta}\left(\beta-\zeta_{1}\right)}}{(1+i) \sqrt{\zeta_{1}}} \int_{\epsilon \rightarrow 0}^{\infty} d|\tilde{p}| \frac{1}{e^{2 \pi|\tilde{p}|}-1} e^{i \frac{|\tilde{p}|}{\beta}\left(\xi_{2}-\xi_{1}\right)} e^{-i \frac{\pi}{\beta}\left[\frac{|\tilde{p}|}{2}+u_{0}^{\prime}[\tilde{p} \mid]\right]\left(\beta-\zeta_{1}\right)} \times \\
& \frac{J_{-i|\tilde{p}|}\left(\frac{2}{\beta} \sqrt{l^{2}+l+1} \zeta_{2}\right)}{J_{-i|\tilde{p}|}\left(-2 i \sqrt{l^{2}+l+1}\right)} \int_{0}^{\infty} d w \frac{e^{-i \frac{\pi}{\beta} w\left(\beta-\zeta_{1}\right)}}{-\pi^{2}\left(u_{0}^{\prime 2}[|\tilde{p}|]+w^{2}\right)+4\left(l^{2}+l+1\right)-2 \pi^{2} u_{0}^{\prime}[|\tilde{p}|] w} \\
& +\frac{i}{\beta^{\frac{3}{2}}} \frac{1}{2 \pi} \frac{e^{-i \frac{\pi}{\beta} u_{0}^{\prime}[0]\left(\beta-\zeta_{1}\right)}}{\sqrt{\zeta_{1}}} \int_{\frac{\pi}{2}}^{\frac{3 \pi}{2}} d \theta e^{i \frac{\theta}{2}} e^{-i \frac{3 \pi}{4 \beta} e^{-i \theta\left(\beta-\zeta_{1}\right)}} \frac{I_{0}\left(\frac{2}{\beta} \sqrt{l^{2}+l+1} e^{-i \theta} \zeta_{2}\right)}{I_{0}\left(2 \sqrt{l^{2}+l+1}\right)} \times \\
& \int_{0}^{\infty} d w \frac{e^{-\frac{\pi}{\beta} w e^{-i \theta}\left(\beta-\zeta_{1}\right)}}{\pi^{2}\left(u_{0}^{\prime 2}[0] e^{2 i \theta}-w^{2}\right)+4\left(l^{2}+l+1\right)-2 i \pi^{2} u_{0}^{\prime}[0] e^{i \theta} w}
\end{aligned}
$$

The analysis as to the physical significance of the singular part $D_{a s}\left(x_{2}-x_{1}\right)$ of the Green function in (6) applies identically also to (66) and to (67). In addition, (66) and (67) are single valued and manifestly convergent for any values of $\tau_{i} \in[0,8 \pi M] ; i=1,2$ and for any values of $\rho_{i} ; i=1,2$ in the range within which the present analytical extension of (6) is a valid approximation to the Hartle-Hawking Euclidean Green function in the interior of the Schwarzschild black hole. For that matter, in $1+1$ dimensions the additive result of (66) and (67) is, in the interior of the Schwarzschild black hole, the physically valid analytical extension of the boundary part $D_{b}\left(x_{2}-x_{1}\right)$ of the Green function in (6). The crucial aspect in its derivation has been the demand that the analytical extension of (47) and (48) be such as to, independently, preserve - at each stage of the calculation - the convergent character which these expressions have for all values of $\xi_{2,1}$ and $\zeta_{2,1}$.

\section{Scalar Propagation Inside The Schwarzschild Black Hole}

The results hitherto obtained signify in $1+1$ dimensions a valid approximation to the Hartle-Hawking Euclidean Green function for the massless conformal scalar field in the interior of the Schwarzschild black hole. The associated range of validity will be established in what follows. Within that range the approximation to the HartleHawking Euclidean Green function in the interior of the Schwarzschild black hole in four dimensions will be obtained by the appropriate replacement of (6) in the context of the preceding analytical extensions. Specifically, as a result of (33), (45) and (9) the contribution which the singular part $D_{a s}\left(x_{2}-x_{1}\right)$ of the Euclidean Green function in (6) has to the corresponding Euclidean Green function in the interior is

$$
D_{a s}^{(i n t)}\left(x_{2}-x_{1}\right)=\frac{i}{\beta} \frac{e^{-\frac{3 \pi}{4 \beta}\left(\zeta_{2}-\zeta_{1}\right)}}{\sqrt{\zeta_{1} \zeta_{2}}} \int_{\epsilon \rightarrow 0}^{\infty} d|\tilde{p}| \frac{1}{1-e^{-2 \pi|\tilde{p}|}} e^{i \frac{\tilde{p} \mid}{\beta}\left(\xi_{2}-\xi_{1}\right)} e^{i \frac{\pi}{\beta}\left[\frac{\tilde{p} \mid}{2}+u_{0}[|\tilde{p}|]\right]\left(\zeta_{2}-\zeta_{1}\right)} \times
$$




$$
\begin{aligned}
& \sum_{l=0}^{\infty} \sum_{m=-l}^{l} Y_{l m}\left(i \tilde{\theta}_{2}, \phi_{2}\right) Y_{l m}^{*}\left(i \tilde{\theta}_{1}, \phi_{1}\right) \int_{0}^{\infty} d w \frac{e^{-i \frac{\pi}{\beta} w\left(\zeta_{2}-\zeta_{1}\right)}}{\pi^{2}\left(u_{0}[|\tilde{p}|]-w\right)^{2}-4\left(l^{2}+l+1\right)} \\
& +\frac{i}{\beta} \frac{e^{\frac{3 \pi}{4 \beta}\left(\zeta_{2}-\zeta_{1}\right)}}{\sqrt{\zeta_{1} \zeta_{2}}} \int_{\epsilon \rightarrow 0}^{\infty} d|\tilde{p}| \frac{1}{1-e^{-2 \pi|\tilde{p}|}} e^{i \frac{|\tilde{p}|}{\beta}\left(\xi_{2}-\xi_{1}\right)} e^{i \frac{\pi}{\beta}\left[\frac{|\tilde{p}|}{2}+u_{0}[|\tilde{p}|]\right]\left(\zeta_{2}-\zeta_{1}\right)} \times \\
& \sum_{l=0}^{\infty} \sum_{m=-l}^{l} Y_{l m}\left(i \tilde{\theta}_{2}, \phi_{2}\right) Y_{l m}^{*}\left(i \tilde{\theta}_{1}, \phi_{1}\right) \int_{0}^{\infty} d w \frac{e^{i \frac{\pi}{\beta} w\left(\zeta_{2}-\zeta_{1}\right)}}{\pi^{2}\left(u_{0}[|\tilde{p}|]+w\right)^{2}-4\left(l^{2}+l+1\right)} \\
& +\frac{i}{\beta} \frac{1}{2 \pi} \frac{e^{i \frac{\pi}{\beta} u_{0}[0]\left(\zeta_{2}-\zeta_{1}\right)}}{\sqrt{\zeta_{1} \zeta_{2}}} \int_{\frac{\pi}{2}}^{\frac{3 \pi}{2}} d \theta e^{i \theta} e^{i \frac{3 \pi}{4 \beta} e^{-i \theta}\left(\zeta_{2}-\zeta_{1}\right)} \times \\
& \sum_{l=0}^{\infty} \sum_{m=-l}^{l} Y_{l m}\left(i \tilde{\theta}_{2}, \phi_{2}\right) Y_{l m}^{*}\left(i \tilde{\theta}_{1}, \phi_{1}\right) \int_{0}^{\infty} d w \frac{e^{-\frac{\pi}{\beta} w e^{-i \theta}\left(\zeta_{2}-\zeta_{1}\right)}}{\pi^{2}\left(u_{0}[0] e^{i \theta}+i w\right)^{2}+4\left(l^{2}+l+1\right)} \\
& -\frac{i}{\beta} \frac{e^{\frac{3 \pi}{4 \beta}\left(\zeta_{2}-\zeta_{1}\right)}}{\sqrt{\zeta_{1} \zeta_{2}}} \int_{\epsilon \rightarrow 0}^{\infty} d|\tilde{p}| \frac{1}{1-e^{-2 \pi|\tilde{p}|}} e^{i \frac{|\tilde{p}|}{\beta}\left(\xi_{2}-\xi_{1}\right)} e^{-i \frac{\pi}{\beta}\left[\frac{\mid \tilde{p}}{2}+u_{0}[|\tilde{p}|]\right]\left(\zeta_{2}-\zeta_{1}\right)} \times \\
& \sum_{l=0}^{\infty} \sum_{m=-l}^{l} Y_{l m}\left(i \tilde{\theta}_{2}, \phi_{2}\right) Y_{l m}^{*}\left(i \tilde{\theta}_{1}, \phi_{1}\right) \int_{0}^{\infty} d w \frac{e^{-i \frac{\pi}{\beta} w\left(\zeta_{2}-\zeta_{1}\right)}}{\pi^{2}\left(u_{0}[|\tilde{p}|]+w\right)^{2}-4\left(l^{2}+l+1\right)} \\
& -\frac{i}{\beta} \frac{e^{-\frac{3 \pi}{4 \beta}\left(\zeta_{2}-\zeta_{1}\right)}}{\sqrt{\zeta_{1} \zeta_{2}}} \int_{\epsilon \rightarrow 0}^{\infty} d|\tilde{p}| \frac{1}{1-e^{-2 \pi|\tilde{p}|}} e^{i \frac{|\tilde{p}|}{\beta}\left(\xi_{2}-\xi_{1}\right)} e^{-i \frac{\pi}{\beta}\left[\frac{\tilde{p} \mid}{2}+u_{0}[|\tilde{p}|]\right]\left(\zeta_{2}-\zeta_{1}\right)} \times \\
& \sum_{l=0}^{\infty} \sum_{m=-l}^{l} Y_{l m}\left(i \tilde{\theta}_{2}, \phi_{2}\right) Y_{l m}^{*}\left(i \tilde{\theta}_{1}, \phi_{1}\right) \int_{0}^{\infty} d w \frac{e^{i \frac{\pi}{\beta} w\left(\zeta_{2}-\zeta_{1}\right)}}{\pi^{2}\left(u_{0}[|\tilde{p}|]-w\right)^{2}-4\left(l^{2}+l+1\right)} \\
& -\frac{i}{\beta} \frac{1}{2 \pi} \frac{e^{-i \frac{\pi}{\beta} u_{0}[0]\left(\zeta_{2}-\zeta_{1}\right)}}{\sqrt{\zeta_{1} \zeta_{2}}} \int_{\frac{\pi}{2}}^{\frac{3 \pi}{2}} d \theta e^{i \theta} e^{-i \frac{3 \pi}{4 \beta} e^{-i \theta}\left(\zeta_{2}-\zeta_{1}\right)} \sum_{l=0}^{\infty} \sum_{m=-l}^{l} Y_{l m}\left(i \tilde{\theta}_{2}, \phi_{2}\right) Y_{l m}^{*}\left(i \tilde{\theta}_{1}, \phi_{1}\right) \times \\
& \int_{0}^{\infty} d w \frac{e^{-\frac{\pi}{\beta} w e^{-i \theta}\left(\zeta_{2}-\zeta_{1}\right)}}{\pi^{2}\left(u_{0}[0] e^{i \theta}-i w\right)^{2}+4\left(l^{2}+l+1\right)}
\end{aligned}
$$

Inherent in (68) is the expected quadratic divergence at $x_{2} \rightarrow x_{1}$ which will be analyzed in what follows.

Likewise, as a result of (66), (67) and (9) the contribution which the boundary part $D_{b}\left(x_{2}-x_{1}\right)$ of the Euclidean Green function in (6) has, in four dimensions, to the corresponding Euclidean Green function in the interior of the Schwarzschild black hole is

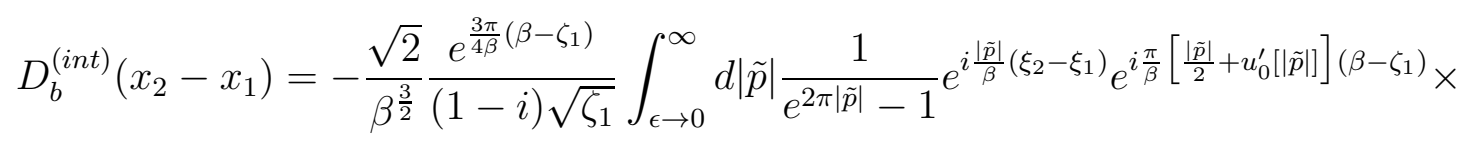




$$
\begin{aligned}
& \sum_{l=0}^{\infty} \sum_{m=-l}^{l} Y_{l m}\left(i \tilde{\theta}_{2}, \phi_{2}\right) Y_{l m}^{*}\left(i \tilde{\theta}_{1}, \phi_{1}\right) \frac{J_{i|\tilde{p}|}\left(-\frac{2}{\beta} \sqrt{l^{2}+l+1} \zeta_{2}\right)}{J_{i|\tilde{p}|}\left(-2 i \sqrt{l^{2}+l+1}\right)} \int_{0}^{\infty} d w \frac{e^{i \frac{\pi}{\beta} w\left(\beta-\zeta_{1}\right)}}{\pi^{2}\left(u_{0}^{\prime}[|\tilde{p}|]+w\right)^{2}-4\left(l^{2}+l+1\right)} \\
& +\frac{\sqrt{2}}{\beta^{\frac{3}{2}}} \frac{e^{-\frac{3 \pi}{4 \beta}\left(\beta-\zeta_{1}\right)}}{(1+i) \sqrt{\zeta_{1}}} \int_{\epsilon \rightarrow 0}^{\infty} d|\tilde{p}| \frac{1}{e^{2 \pi|\tilde{p}|}-1} e^{i \frac{|\tilde{p}|}{\beta}\left(\xi_{2}-\xi_{1}\right)} e^{i \frac{\pi}{\beta}\left[\frac{|\tilde{p}|}{2}+u_{0}^{\prime}[|\tilde{p}|]\right]\left(\beta-\zeta_{1}\right)} \times \\
& \sum_{l=0}^{\infty} \sum_{m=-l}^{l} Y_{l m}\left(i \tilde{\theta}_{2}, \phi_{2}\right) Y_{l m}^{*}\left(i \tilde{\theta}_{1}, \phi_{1}\right) \frac{J_{-i|\tilde{p}|}\left(\frac{2}{\beta} \sqrt{l^{2}+l+1} \zeta_{2}\right)}{J_{-i|\tilde{p}|}\left(-2 i \sqrt{l^{2}+l+1}\right)} \int_{0}^{\infty} d w \frac{e^{-i \frac{\pi}{\beta} w\left(\beta-\zeta_{1}\right)}}{\pi^{2}\left(u_{0}^{\prime}[|\tilde{p}|]-w\right)^{2}-4\left(l^{2}+l+1\right)} \\
& -\frac{i}{\beta^{\frac{3}{2}}} \frac{1}{2 \pi} \frac{e^{i \frac{\pi}{\beta} u_{0}^{\prime}[0]\left(\beta-\zeta_{1}\right)}}{\sqrt{\zeta_{1}}} \int_{\frac{\pi}{2}}^{\frac{3 \pi}{2}} d \theta e^{i \frac{\theta}{2}} e^{i \frac{3 \pi}{4 \beta} e^{-i \theta}\left(\beta-\zeta_{1}\right)} \times \\
& \sum_{l=0}^{\infty} \sum_{m=-l}^{l} Y_{l m}\left(i \tilde{\theta}_{2}, \phi_{2}\right) Y_{l m}^{*}\left(i \tilde{\theta}_{1}, \phi_{1}\right) \frac{I_{0}\left(\frac{2}{\beta} \sqrt{l^{2}+l+1} e^{-i \theta} \zeta_{2}\right)}{I_{0}\left(2 \sqrt{l^{2}+l+1}\right)} \int_{0}^{\infty} d w \frac{e^{-\frac{\pi}{\beta} w e^{-i \theta}\left(\beta-\zeta_{1}\right)}}{\pi^{2}\left(u_{0}^{\prime}[0] e^{i \theta}+i w\right)^{2}+4\left(l^{2}+l+1\right)} \\
& +\frac{\sqrt{2}}{\beta^{\frac{3}{2}}} \frac{e^{-\frac{3 \pi}{4 \beta}\left(\beta-\zeta_{1}\right)}}{(1-i) \sqrt{\zeta_{1}}} \int_{\epsilon \rightarrow 0}^{\infty} d|\tilde{p}| \frac{1}{e^{2 \pi|\tilde{p}|}-1} e^{i \frac{|\tilde{p}|}{\beta}\left(\xi_{2}-\xi_{1}\right)} e^{-i \frac{\pi}{\beta}\left[\frac{|\tilde{p}|}{2}+u_{0}^{\prime}[|\tilde{p}|]\right]\left(\beta-\zeta_{1}\right)} \times \\
& \sum_{l=0}^{\infty} \sum_{m=-l}^{l} Y_{l m}\left(i \tilde{\theta}_{2}, \phi_{2}\right) Y_{l m}^{*}\left(i \tilde{\theta}_{1}, \phi_{1}\right) \frac{J_{i|\tilde{p}|}\left(-\frac{2}{\beta} \sqrt{l^{2}+l+1} \zeta_{2}\right)}{J_{i|\tilde{p}|}\left(-2 i \sqrt{l^{2}+l+1}\right)} \int_{0}^{\infty} d w \frac{e^{i \frac{\pi}{\beta} w\left(\beta-\zeta_{1}\right)}}{\pi^{2}\left(u_{0}^{\prime}[|\tilde{p}|]-w\right)^{2}-4\left(l^{2}+l+1\right)} \\
& -\frac{\sqrt{2}}{\beta^{\frac{3}{2}}} \frac{e^{\frac{3 \pi}{4 \beta}\left(\beta-\zeta_{1}\right)}}{(1+i) \sqrt{\zeta_{1}}} \int_{\epsilon \rightarrow 0}^{\infty} d|\tilde{p}| \frac{1}{e^{2 \pi|\tilde{p}|}-1} e^{i \frac{|\tilde{p}|}{\beta}\left(\xi_{2}-\xi_{1}\right)} e^{-i \frac{\pi}{\beta}\left[\frac{|\tilde{p}|}{2}+u_{0}^{\prime}[|\tilde{p}|]\right]\left(\beta-\zeta_{1}\right)} \times \\
& \sum_{l=0}^{\infty} \sum_{m=-l}^{l} Y_{l m}\left(i \tilde{\theta}_{2}, \phi_{2}\right) Y_{l m}^{*}\left(i \tilde{\theta}_{1}, \phi_{1}\right) \frac{J_{-i|\tilde{p}|}\left(\frac{2}{\beta} \sqrt{l^{2}+l+1} \zeta_{2}\right)}{J_{-i|\tilde{p}|}\left(-2 i \sqrt{l^{2}+l+1}\right)} \int_{0}^{\infty} d w \frac{e^{-i \frac{\pi}{\beta} w\left(\beta-\zeta_{1}\right)}}{\pi^{2}\left(u_{0}^{\prime}[|\tilde{p}|]+w\right)^{2}-4\left(l^{2}+l+1\right)} \\
& +\frac{i}{\beta^{\frac{3}{2}}} \frac{1}{2 \pi} \frac{e^{-i \frac{\pi}{\beta} u_{0}^{\prime}[0]\left(\beta-\zeta_{1}\right)}}{\sqrt{\zeta_{1}}} \int_{\frac{\pi}{2}}^{\frac{3 \pi}{2}} d \theta e^{i \frac{\theta}{2}} e^{-i \frac{3 \pi}{4 \beta} e^{-i \theta}\left(\beta-\zeta_{1}\right)} \times \\
& \sum_{l=0}^{\infty} \sum_{m=-l}^{l} Y_{l m}\left(i \tilde{\theta}_{2}, \phi_{2}\right) Y_{l m}^{*}\left(i \tilde{\theta}_{1}, \phi_{1}\right) \frac{I_{0}\left(\frac{2}{\beta} \sqrt{l^{2}+l+1} e^{-i \theta} \zeta_{2}\right)}{I_{0}\left(2 \sqrt{l^{2}+l+1}\right)} \times \\
& \int_{0}^{\infty} d w \frac{e^{-\frac{\pi}{\beta} w e^{-i \theta}\left(\beta-\zeta_{1}\right)}}{\pi^{2}\left(u_{0}^{\prime}[0] e^{i \theta}-i w\right)^{2}+4\left(l^{2}+l+1\right)}
\end{aligned}
$$

The expressions in (68) and (69) constitute the central result of this project's entire analysis. Away from the coincidence space-time limit each of these two expressions is a regular function of the space-time coordinates. In addition, at $x_{2} \rightarrow x_{1}$ the expression in (69) remains regular whereas, as is analyzed below, the expression in (68) features the expected quadratic divergence. Consequently, within the corresponding range of validity the approximation to the Hartle-Hawking Euclidean Green function for the massless 
conformal scalar field in the interior of the Schwarzschild black hole emerges as the additive result of (68) and (69). It should be obvious that, although invariably finite for all values of $\zeta_{1,2}>0$, the two terms which correspond to $|\tilde{p}| \rightarrow 0$ in $D_{a s}^{(i n t)}\left(x_{2}-x_{1}\right)$ are necessary for the single-valued character of that function. The absorption of these two terms in the invariably finite $D_{b}^{(i n t)}\left(x_{2}-x_{1}\right)$ would result in an inconsistent expression for the asymptotic part of the Green function. Consequently, always within the associated range of validity, the contribution $D_{a s}^{(i n t)}\left(x_{2}-x_{1}\right)$ cited in (68) coincides with the singular part of the Hartle-Hawking Euclidean Green function for the massless conformal scalar field in the interior of the Schwarzschild black hole. For that matter, the contribution $D_{b}^{(i n t)}\left(x_{2}-x_{1}\right)$ cited in (69) coincides, itself, with the boundary part of that Green function.

The fact that, at the coincidence space-time limit $x_{2} \rightarrow x_{1}$, the dominant contribution to $D_{\text {as }}^{(i n t)}\left(x_{2}-x_{1}\right)$ emerges from the ultra-violet domain $|\tilde{p}| \rightarrow \infty$ and $w \rightarrow \infty$ in transfer space implies at once that, at $x_{2} \rightarrow x_{1}$, the dominant contribution to the integral over $w$ comes from $w>>l$ in each term in the series over $l$. Consequently, the completeness relation [8]

$$
\sum_{l=0}^{\infty} \sum_{m=-l}^{l} Y_{l m}\left(z_{2}, \phi_{2}\right) Y_{l m}^{*}\left(z_{1}, \phi_{1}\right)=\delta\left(z_{2}-z_{1}\right) \delta\left(\phi_{2}-\phi_{1}\right) ; z_{i} \in C, i=1,2
$$

and the expansion

$$
\delta\left(\cosh \theta_{2}-\cosh \theta_{1}\right) \delta\left(\phi_{2}-\phi_{1}\right)=\frac{1}{4 \pi} \sum_{k=0}^{\infty}(2 k+1) P_{k}(\cosh \gamma)
$$

with

$$
\cosh \gamma=\cosh \theta_{2} \cosh \theta_{1}-\sinh \theta_{2} \sinh \theta_{1} \cos \left(\phi_{2}-\phi_{1}\right)
$$

imply by dint of power counting that at $x_{2} \rightarrow x_{1}$ each of the first two terms on the right side of the expressions in (33) and (45) respectively are, independently, quadratically divergent. This fact, however, does not by itself imply the presence of a quadratic divergence at $x_{2} \rightarrow x_{1}$ in (68). In fact, since (33) and (45) are independently defined for all values of $\zeta_{2}$ and $\zeta_{1}$ within the associated range of validity it follows that, as a consequence of the entire analytical procedure, the asymptotic propagator in (68) is formally indeterminate at $x_{2} \rightarrow x_{1}$. In order to render concrete meaning to the expression in (68) at $x_{2} \rightarrow x_{1}$ use must, instead, be made of the fact that the additive result of (33) and (45) is an equivalent representation of the expression in (10) for purely imaginary values of $\rho_{1,2}$. The significance of this fact was analyzed in the context of (33). As a direct consequence, (68) features, at $x_{2} \rightarrow x_{1}$, the same divergence structure as that inherent in (10). In view of [4] it is

$$
D_{a s}^{(i n t)}\left(x_{2} \rightarrow x_{1}\right)=\frac{1}{2 \pi^{2} \beta^{2}} \sum_{k=0}^{\infty}(2 k+1)\left[\sum_{p=0}^{p_{0}>>1} \frac{1}{v_{p}(\zeta)}+\frac{1}{c} \sum_{p=p_{0}}^{\infty} \frac{1}{p}\right]+F(\zeta)
$$


with $c>1$ and with $v_{p}(\zeta)>1$ and $F(\zeta)$ being arbitrary, smooth functions which remain finite throughout their corresponding domains and such that $v_{p}(\zeta) \approx c p$, if $p>>1$. The expression in (72) explicitly manifests the quadratic divergence expected of the asymptotic propagator in four dimensions at the coincidence space-time limit in the interior of the black hole. In conformity with (70) and (71) that quadratic divergence also characterises, at $x_{2} \rightarrow x_{1}$, the expressions in (33) and (45) respectively. In real time the same divergence will, of course, also occur when two distinct points $x_{2}$ and $x_{1}$ can be joined by a null geodesic. The quadratic divergence inherent in (72) is consistent with theoretical expectations of a scalar propagator on any manifold.

The analysis hitherto accomplished has yielded, in $1+1$ and in four dimensions respectively, a mathematical expression which is a valid approximation to the Hartle-Hawking Euclidean Green function for a massless conformal scalar field within a certain range of values of the radial coordinate $r$ in the interior of the Schwarzschild black hole. In order to assess that range of values within which the additive result $D^{(i n t)}\left(x_{2}-x_{1}\right)$ of the expressions in (68) and (69) is the stated valid approximation it must be recalled that - as stated in Sec.III - in terms of the Schwarzschild radial variable $r$ the maximum possible range of validity for the analytical extension of $D\left(x_{2}-x_{1}\right)$ in (6) in the interior of the black hole is $(0, M)$ since it is only that range which conforms with (34). However, since the condition in (21) has been imposed only on those values of $\rho$ for which (6) is valid the stated range of validity in the interior is necessarily restricted by (7) and (8) respectively. In effect, as a result of (34), the range of validity for the analytical extension accomplished in the additive result of (68) and (69) is determined by

$$
0<\left|\rho_{\text {int }}\right|^{2} \leq \frac{\beta^{2}}{100}
$$

and accordingly, in terms of $r$, by

$$
1.980 M \leq r \leq 2 M
$$

This range of values below the event horizon signifies for the validity of the approximation to the exact propagator the same order of magnitude as that expressed in (8) above the event horizon.

As was also the case with the conformal scalar propagator derived in [4] in the exterior region of the Schwarzschild black-hole geometry the result

$$
D^{(i n t)}\left(x_{2}-x_{1}\right)=D_{a s}^{(i n t)}\left(x_{2}-x_{1}\right)+D_{b}^{(i n t)}\left(x_{2}-x_{1}\right)
$$

- with $D_{a s}^{(i n t)}\left(x_{2}-x_{1}\right)$ and $D_{b}^{(i n t)}\left(x_{2}-x_{1}\right)$ respectively given in (68) and (69) - signifies the only approximation which specifically and without a priori assumptions expresses the massless conformal scalar propagator in the Hartle-Hawking vacuum state in terms of its space-time dependence in a specific segment of the interior region of the Schwarzschild black-hole geometry. That segment is specified in (73) and, equivalently, in (74).

\section{Scalar Propagator and Black-Hole Radiation}

Having obtained the scalar propagator as an explicitly analytic expression of spacetime in the exterior and interior region of the Schwarzschild black-hole respectively it 
is desirable to extract its inherent information as to the vacuum activity in the HartleHawking state. At first sight it would appear that the expression in (75) is far too complicated for any calculation in that direction. Appearances are - nevertheless - deceptive, as I shall demonstrate in this section. The first comment which must be made in this respect is that such a complication was expected from the outset. The exterior geometry is spherically symmetric and, by dint of Birkhoff's theorem, also static. The time translational invariance which the exterior region has with respect to the Schwarzschild time coordinate $t$ is expressed by its characteristic global time-like Killing vector $\frac{\partial}{\partial t}$. In addition, in the exterior region the vector $\frac{\partial}{\partial r}$ is space-like. In the interior region, however, the Killing vector $\frac{\partial}{\partial t}$ is space-like whereas the vector $\frac{\partial}{\partial r}$ is time-like. This situation is an expression of the fact that in the interior region of the Schwarzschild black hole the roles of time and space are reversed. As a consequence, the interior space-time geometry is dynamic and characterised by the absence of spherical symmetry. The strong contrast between the substantially more complicated expression in (75) and that in (6) is a consequence of the stated difference between the exterior region and the interior region of the Schwarzschild black hole.

The massless conformal scalar propagator in (6) has already yielded a prediction regarding the nature of quantum propagation in the exterior region of the Schwarzschild space-time. In [4] it was shown that the Green function in (6) vanishes at the limit $\rho_{2} \rightarrow 0$ when $\rho_{1} \neq 0$. As stated in the relevant analysis the vanishing effect expressed by

$$
\lim _{\rho_{2} \rightarrow 0} D\left(x_{2}-x_{1}\right)=0 ; \rho_{1} \neq 0
$$

is a consequence of the causal structure of the Schwarzschild black-hole space-time. At the semi-classical limit $\hbar \rightarrow 0$ all observers in the static region, regardless of their state of motion or choice of coordinates, agree that no particle reaches the hole's event horizon within a finite advance of their proper time. Equivalently, at the semi-classical limit all observers in the static region agree that the frequency of a waveform tends to zero in the vicinity of the hole's event horizon. As a consequence, away from the semi-classical limit the transition amplitude for quantum propagation specified by one end-point of propagation being arbitrarily close to the event horizon is also expected to vanish for all observers in the static region.

A naive approach to the physical essence of (76) could eventuate in the conclusion that the latter contradicts the production of particles by the Schwarzschild black hole. For, if the vacuum is that of the Hartle-Hawking state, how can an observer who accelerates to remain stationary at a certain value of the Schwarzschild radial coordinate $r$ register any particles propagating from the vicinity of the event horizon when such a propagation tends to vanish as a consequence of (76)?

The Penrose diagram in Fig.6 describes the essence of the matter. The production of particles by the Schwarzschild black hole arises because the presence of the event horizon renders the Hartle-Hawking vacuum state unstable. The instability of the HartleHawking vacuum and the associated radiation produced by the Schwarzschild black hole can be conceptualised by the production and subsequent annihilation of a virtual particle-antiparticle pair as an expression of vacuum fluctuations. The presence of an event horizon allows for the absence of the subsequent annihilation in the event that 
one of the two particles has already crossed that horizon. Since that particle is, in the interior, necessarily in a quantum state of negative energy with respect to spatial infinity the other particle can transit to arbitrarily large radial distances and be detected as an actual particle in a positive energy state by an observer situated anywhere in the static region of the Schwarzschild space-time. I should add that this largely heuristic approach to Hawking radiation does not define the Hartle-Hawking vacuum state. It is, instead, a consequence of that state's instability.

The static observer whose world line is represented in Fig.6 is situated at a radial distance $r_{1}^{\prime}$ from the event horizon which falls within the range stated in (8). The shaded segment of the Penrose diagram must be replaced by the space-time geometry in the interior of the spherical distribution of matter which undergoes complete gravitational collapse. An equivalent description of the heuristic approach cited above is represented in the Penrose diagram of Fig.6. A quantum particle emerges at some point $B$ of the future singularity, transits as a positive energy state backward in time across the future event horizon $\mathrm{H}^{+}$where it is scattered by the background curvature at some point $C$ in the vicinity of the latter and continues its transition forward in time always as a positive energy state before it registers its existence in the detector of the observer at space-time point $A \equiv\left(t_{1}^{\prime}, r_{1}^{\prime}, \theta_{1}^{\prime}, \phi_{1}^{\prime}\right)$.

The line $B C A$ represents the quantum propagation as described above. The syncopated segment $B Z$ represents quantum propagation at radial values below the lower bound $r_{1}=1.980 M$ in (74). The segment which is depicted by the continuous line from $Z \equiv\left(t_{1}, r_{1}, \theta_{1}, \phi_{1}\right)$ to some point on the future event horizon represents the propagator in the range of validity stated in (74). Consequently, the segment $Z C A$ represents the massless conformal scalar propagator which is described by (75) and (6) in the interior and the exterior region of the Schwarzschild black hole respectively.

The probability for the emergence and transition of the quantum particle from point $B$ on the future singularity to the observation point $A$ is the square of the magnitude of the transition amplitude from $B$ to $A$. That transition amplitude itself is the functional integral

$$
W=\int \mathcal{D}[\Phi] e^{\frac{i}{\hbar} S}
$$

on condition that the Hartle-Hawking vacuum state of the field $\Phi(x)$ has been excited only to the stated quantum particle. The transition amplitude in (77) then is the sum over all paths which emerge at point $B$ and eventuate at point $A$. The action for the massless conformal scalar field is

$$
S=\frac{1}{2} \int d^{4} x[-g(x)]^{\frac{1}{2}}\left[g^{\mu \nu}(x) \partial_{\mu} \Phi(x) \partial_{\nu} \Phi(x)-\frac{1}{6} R(x) \Phi^{2}(x)\right]
$$

with the Schwarzschild metric $g_{\mu \nu}(x)$ expressed in Kruskal coordinates. In the context of (77) the action functional $S$ corresponds to the stated set of paths. In the general case which involves propagation of a field configuration between the future singularity at $r=0$ and the future infinity $\mathcal{J}^{+}$the integral which defines the action functional in (78) is taken over the entire Schwarzschild black-hole space-time (the set of points which 


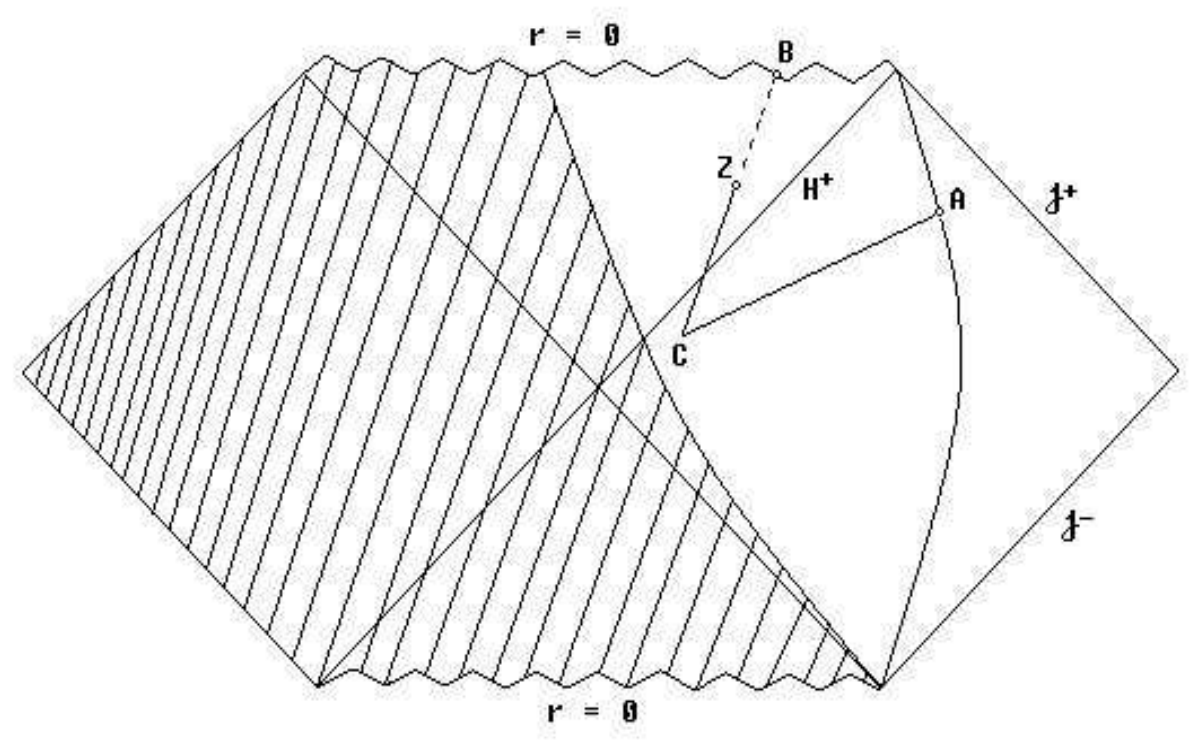

Figure 6. Penrose diagram for complete gravitational collapse of a spherically symmetric distribution of matter. The unshaded segment represents the geometry outside that distribution. The path $B C A$ corresponds to particle production by the Schwarzschild black hole in the Hartle-Hawking vacuum state. The segment $Z C A$ corresponds to radial distances from the future event horizon $H^{+}$within which the propagator in (6) and (75) coincides with the exact massless scalar propagator. The fixed radial distance of the observer whose world line is also represented in the diagram lies within the range of that coincidence.

defines the future singularity is not part of space-time). As the Schwarzschild metric is regular on the event horizon when expressed in Kruskal coordinates the integral over the entire space-time is rigorously defined.

The measure $\mathcal{D}[\Phi]$ in the path integral does not include paths which emerge from past null infinity $\mathcal{J}^{-}$as such paths represent the propagation of incoming particles from the infinite past. Neither does the measure include paths which pass through the shaded segment of the Penrose diagram since, as stated, that region must be replaced by the space-time geometry in the interior of the spherical distribution of matter which collapses to a black hole and will not contribute to the production of particles at late times [9].

The path integral which has thus been formally defined can be given concrete meaning through the analytic continuation of the action to values which result in integration over an exponentially damped functional in (77). This can be accomplished by continuing the 
Kruskal coordinates to a domain in which the Schwarzschild metric has signature +4 . However, as the objective in this section is to demonstrate the relation of the propagator in (75) and (6) to Hawking radiation it is necessary to return to the coordinates in which the metric receives the expression in (9) and (4) in the interior and the exterior region respectively, with $\tau$ identified as an angular variable of period $8 \pi M$. In the Euclidean Schwarzschild manifold which emerges as a result of this arrangement the interior spacetime point $Z$ in Fig. 6 corresponds to the set $\left(i \xi_{1}, i \zeta_{1}, i \theta_{1}, \phi_{1}\right)$ and the exterior space-time point $A$ to $\left(\tau_{1}^{\prime}, \rho_{1}^{\prime}, \theta_{1}^{\prime}, \phi_{1}^{\prime}\right)$. The future event horizon $(r=2 M)$ in Fig.6 corresponds, on the Euclidean Schwarzschild manifold, to a two-dimensional sphere of coordinate values $(i \zeta=\rho=0, \theta, \phi)$. The point on the future event horizon which, in Fig.6, is common to scalar propagation in the exterior region and in the interior region of the black hole corresponds to a point which is common to scalar propagation in (75) and (6) with coordinates $\left(i \zeta_{2}=\rho_{2}=0, \theta_{2}, \phi_{2}\right)$.

In referring to the Euclidean Schwarzschild manifold it is important to remember that the latter is the union of the Euclidean Schwarzschild manifold for $r \geq 2 M$ on which the Green function in (6) was obtained and of the Euclidean Schwarzschild manifold for $r \leq 2 M$ on which the Green function in (75) was obtained. As the Schwarzschild coordinates are singular on the event horizon the coordinate transformation in (2) implies that - with the angular dependence on $\theta$ and $\phi$ suppressed - the two manifolds are connected only at the point $\rho=0$ - or, equivalently, at $r=2 M$.

If the vanishing effect expressed in (76) is to be valid in the event that scalar propagation occurs exclusively in the static region toward or from the vicinity of the event horizon but not in the event of scalar propagation which also extends in the interior region of the Schwarzschild black hole then, necessarily, that vanishing effect - being a property of scalar propagation exclusively in the exterior region - must be offset by a property of scalar propagation which exists exclusively in the interior of the black hole. In non-rigorous language, we expect the uncertainty principle - which is heuristically the cause of Hawking radiation in the Hartle-Hawking vacuum state - to act in such a way as to cancel the vanishing effect expressed in (76). In what follows I shall show that this is, indeed, the case.

As, in Fig.6, the Hartle-Hawking vacuum state for the massless scalar field has been excited to one particle whose position at $i \xi_{1}$ and $\tau_{1}^{\prime}$ has been respectively specified to be the point corresponding to $Z$ and to $A$ the amplitude $<\Phi_{1}^{\prime}, \tau_{1}^{\prime} \mid \Phi_{1}, i \xi_{1}>$ for field configuration $\Phi_{1}$ at $i \xi_{1}$ to propagate, on the Euclidean Schwarzschild manifold, to field configuration $\Phi_{1}^{\prime}$ at $\tau_{1}^{\prime}$ is identical to the transition amplitude $<\rho_{1}^{\prime}, \theta_{1}^{\prime}, \phi_{1}^{\prime}, \tau_{1}^{\prime} \mid i \zeta_{1}, i \theta_{1}, \phi_{1}, i \xi_{1}>$. This, in turn, relates directly to quantum propagation between space-time points $Z$ and $A$ on the manifold of Fig.6. On the Euclidean Schwarzschild manifold for $r \leq 2 M$ the propagator which expresses the transition amplitude $<i \zeta_{2}=0, \theta_{2}, \phi_{2}, \mid i \zeta_{1}, i \theta_{1}, \phi_{1}, i \xi_{1}>$ is a functional integral over the paths which connect the point associated with $Z$ to the common point which is specified on the two-sphere at $i \zeta_{2}=0(r=2 M)$ by $\theta_{2}$ and $\phi_{2}$. That functional integral is the Euclidean version of the path integral in (77) with the action in (78) defined on the Euclidean Schwarzschild manifold for $r \leq 2 M$ and with the Schwarzschild metric $g_{\mu \nu}(x)$ being expressed by (9) in the context of (2). It coincides, therefore, with the propagator in (75) if $x_{1}=\left(i \zeta_{1}, i \theta_{1}, \phi_{1}, i \xi_{1}\right)$ and $x_{2}=\left(i \zeta_{2}=0, \theta_{2}, \phi_{2}\right)$. At once, the 
propagator which on the Euclidean Schwarzschild manifold for $r \geq 2 M$ expresses the transition amplitude $<\rho_{1}^{\prime}, \theta_{1}^{\prime}, \phi_{1}^{\prime}, \tau_{1}^{\prime} \mid i \zeta_{2}=\rho_{2}=0, \theta_{2}, \phi_{2}>$ is a functional integral over the paths which connect the common point on the two-sphere at $\rho_{2}=0(r=2 M)$ to the point associated with $A$. That functional integral is, again, the Euclidean version of the path integral in (77) with the action in (78) defined on the Euclidean Schwarzschild manifold for $r \geq 2 M$ and with the Schwarzschild metric being expressed by (4). It coincides, therefore, with the propagator in (6) if $x_{1}=\left(\rho_{1}^{\prime}, \theta_{1}^{\prime}, \phi_{1}^{\prime}, \tau_{1}^{\prime}\right)$ and $x_{2}=\left(\rho_{2}=0, \theta_{2}, \phi_{2}\right)$.

This allows for the match of the two transition amplitudes across the future event horizon $H^{+}$. In general, the transition amplitude $<\Phi_{2}, t_{2} \mid \Phi_{1}, t_{1}>$ is the superposition

$$
<\Phi_{2}, t_{2}\left|\Phi_{1}, t_{1}>=\int \mathcal{D} \Phi_{t_{0}}<\Phi_{2}, t_{2}\right| \Phi_{t_{0}}, t_{0}><\Phi_{t_{0}}, t_{0} \mid \Phi_{1}, t_{1}>
$$

with respect to a complete set of modes $\left\{\mid \Phi_{t_{0}}, t_{0}>\right\}$ defined on a space-like hypersurface which corresponds to some $t_{0} \in\left[t_{1}, t_{2}\right]$ and with $\mathcal{D} \Phi_{t_{0}}$ being the corresponding measure over all field configurations defined on that hypersurface. A necessary and sufficient condition for (79) is that the space-time be globally hyperbolic with the space-like hypersurface being, at once, a Cauchy hypersurface. If the hypersurface across which the transition amplitudes in (79) are matched is null, as is the case with the event horizon, then the situation is substantially more involved. Although, in the case of a massless field, the past infinity $\mathcal{J}^{-}$is a Cauchy hypersurface neither is the future infinity $\mathcal{J}^{+}$ nor is the future event horizon $\mathrm{H}^{+}$respectively a Cauchy hypersurface for the collapse space-time in Fig.6. In the case of a massless field only the union of these two null hypersurfaces is a Cauchy hypersurface. Consequently, in the case of a massless field, the modes defined on $\mathrm{H}^{+}$do not form a complete set. A complete set of modes is defined only on the stated union $H^{+} \cup \mathcal{J}^{+}[10$. That precludes the match of the two transition amplitudes across $\mathrm{H}^{+}$in the collapse space-time. However, as the production of particles by the Schwarzschild black hole is a consequence of the causal and topological structure of space-time - as opposed to, a consequence of the space-time geometry - the transition amplitude between space-time points $Z$ and $A$ in the collapse space-time of Fig.6 coincides with the transition amplitude between the same two points in the maximally extended Kruskal space-time. Mathematically, this may not be directly obvious since the measure $\mathcal{D} \Phi$ in the associated path integral now includes paths which did not exist in the corresponding measure of (77). This change is, nevertheless, offset by the presence of additional field modes in the future event horizon $\mathrm{H}^{+}$of the extended Kruskal manifold. Consequently, quantum propagation can be examined on the maximally extended Kruskal manifold altogether. The advantage of such an approach is that the future event horizon $\mathrm{H}^{+}$of the extended Kruskal manifold is a Cauchy hypersurface and admits, for that matter, a complete set of modes.

As a result, the integration which corresponds to that in (79) will now occur over a complete set of field modes defined on the future event horizon $\mathrm{H}^{+}$of the extended Kruskal manifold. If $\Phi(x)$ is a massless conformal scalar field then in the Euclidean sector of the Schwarzschild metric the transition amplitude in (79) becomes 


$$
<\Phi_{1}^{\prime}, \tau_{1}^{\prime} \mid \Phi_{1}, i \xi_{1}>=\int \mathcal{D} \Phi_{\tau_{2} \rightarrow \infty} \int \mathcal{D}[\Phi] e^{-\frac{1}{\hbar} I\left[\tau_{1}^{\prime}, \tau_{2} \rightarrow \infty\right]} \int \mathcal{D}[\Phi] e^{-\frac{1}{\hbar} I\left[i \xi_{2} \rightarrow i \infty, i \xi_{1}\right]}
$$

In (80) $I\left[i \xi_{2} \rightarrow i \infty, i \xi_{1}\right]$ is the action functional which emerges from the analytical extension of the action functional in (78) to the Euclidean Schwarzschild manifold for $r \leq 2 M$ on condition that integration over $i \xi$ is defined in $\left[i \xi_{1}, i \infty\right)$. The Euclidean action functional $I\left[\tau_{1}^{\prime}, \tau_{2} \rightarrow \infty\right]$ has the corresponding meaning. The temporal coordinate $\tau_{2} \rightarrow \infty$ labels the event horizon at $\rho=0$ which, in turn, corresponds to $H^{+}$in the extended Kruskal manifold . For that matter, it also labels all field configurations on $H^{+}$. The notation $\Phi_{\xi_{2} \rightarrow \infty}$ is equivalent.

In the specific situation which is represented in the collapse Schwarzschild space-time in Fig.6 the scalar field $\Phi(x)$ has been excited to one particle and the objective is the match of the corresponding two scalar propagators in the interior and in the exterior region across $H^{+}$. In view of the preceding analysis, the physical significance of (80) reduces, in such a physical context, to

$$
\int_{-\pi}^{\pi} d \theta_{2} \int_{0}^{2 \pi} d \phi_{2}<\rho_{1}^{\prime}, \theta_{1}^{\prime}, \phi_{1}^{\prime}, \tau_{1}^{\prime}\left|\rho_{2}=0, \theta_{2}, \phi_{2}><i \zeta_{2}=0, \theta_{2}, \phi_{2}\right| i \zeta_{1}, i \theta_{1}, \phi_{1}, i \xi_{1}>
$$

with the complete set of modes defined on the future event horizon $\mathrm{H}^{+}$of the extended Kruskal manifold.

As stated above the explicit expressions of the two transition amplitudes featured in the integrand in (81) are accordingly given by (75) and (6). Since (81) explicitly involves scalar propagation with one end-point specified arbitrarily close to the event horizon for both (75) and (6) it is necessary that the asymptotic behaviour of (75) be also examined before the latter is replaced in (81). The incipient point for such an examination is the condition $\frac{\pi u_{0}[p]}{\beta} \rho_{2}>>p$ stated in (6) for scalar propagation in the exterior region of the Schwarzschild black hole. The latter implies, through (19) and (21), that in the interior region it is

$$
\frac{\pi u_{0}[|\tilde{p}|]}{\beta} \zeta_{2}>>|\tilde{p}|
$$

Clearly, the information which will determine the asymptotic behaviour of the propagator in (75) is inherent in the expression

$$
I\left[u_{0}\right]=\frac{1}{\sqrt{\zeta_{1} \zeta_{2}}} \int_{0}^{\infty} d w \frac{e^{ \pm i \frac{\pi}{\beta} w\left(\zeta_{2}-\zeta_{1}\right)}}{\pi^{2}\left(u_{0}[|\tilde{p}|] \pm w\right)^{2}-4\left(l^{2}+l+1\right)}
$$

which is featured in four of the six terms in (68). Since (68) is defined for all values of $\zeta_{2}$ and $\zeta_{1}$ within the range of validity stated in (73) the limit $\zeta_{2} \rightarrow 0$ can be taken in (68) with $\zeta_{1} \neq 0$. In effect, (82) implies 


$$
\lim _{\zeta_{2} \rightarrow 0} \frac{1}{\sqrt{\zeta_{1} \zeta_{2}}} \int_{0}^{\infty} d w \frac{1}{\pi^{2}\left(w \pm u_{0}\right)^{2}-4\left(l^{2}+l+1\right)}<<\frac{1}{\beta^{2}} \frac{1}{|\tilde{p}|^{2}} \lim _{\Lambda \rightarrow \infty} \lim _{\zeta_{2} \rightarrow 0} \frac{\zeta_{2}^{\frac{3}{2}}}{\sqrt{\zeta_{1}}} \int_{0}^{\Lambda} d w=0
$$

The result in (84) implies directly that the real and the imaginary part of $I\left[u_{0}\right]$ respectively vanish at $\zeta_{2} \rightarrow 0$ on condition that $\zeta_{1} \neq 0$. Consequently, at the same limit each of the stated four terms in (68) vanishes identically. This, shifts the issue of the scalar propagator's behaviour at $\zeta_{2} \rightarrow 0$ on the remaining two terms in (68) - namely, on the third and the sixth term.

The third and the sixth term in (68) correspond to $|\tilde{p}| \rightarrow 0$. As stated, they are invariably finite for $\zeta_{1,2}>0$ and necessary for the single-valued character of $D_{a s}^{(i n t)}\left(x_{2}-\right.$ $\left.x_{1}\right)$. Inspection of the integral over $\omega$ in these two terms reveals that its mathematical structure is substantially different from that of the corresponding integral over $\omega$ in the previous four terms. The reason is that at $|\tilde{p}| \rightarrow 0$ (82) allows for two possibilities regarding $u_{0}[0]$. These are respectively, $u_{0}[0]>>\frac{c}{\zeta_{2}}$ with $c>0 ; \zeta_{2} \neq 0$ and a $\zeta$ independent $u_{0}[0]>0$. This situation should be contrasted against the corresponding situation in the exterior region where the transfer space variable $p$ receives values from the discrete set $0,1,2, \ldots$. In that case, if the positive $u_{0}[0]$ is a $\rho_{2}$-independent constant then, at $\rho_{2} \rightarrow 0$ the left side of the condition $\frac{\pi u_{0}[0]}{\beta} \rho_{2}>>0$ lies invariably in a neighbourhood of $p=0$ as is, for example, the subset $\{0,1\}$. In addition, this is always the case however high the value of the constant $u_{0}[0]$ may be. Consequently, if $u_{0}[0]$ does not depend on $\rho_{2}$, the right side $\frac{\pi u_{0}[0]}{\beta} \rho_{2}$ is not much bigger than zero and the condition $\frac{\pi u_{0}[p]}{\beta} \rho_{2}>>p$ is violated for $p=0$, even though the inequality $\frac{\pi u_{0}[0]}{\beta} \rho_{2}>0$ is valid. In effect, in the exterior region the condition $\frac{\pi u_{0}[p]}{\beta} \rho_{2}>>p$ implies exclusively that $u_{0}[0]>>\frac{c}{\rho_{2}}$ with $c>1 ; \rho_{2} \neq 0$. Put simply, in the exterior region $u_{0}[0]$ can not - in $\frac{\pi u_{0}[0]}{\beta} \rho_{2}>>0$ - be a $\rho_{2}$-independent constant because the value $p=0$ can always be replaced by $p=0+1$ [4].

Not so in the interior region where the transfer-space variable $|\tilde{p}|$ receives values in $[0,+\infty)$. In that case a positive $\zeta_{2}$-independent $u_{0}[0]$ is admissible since at $\zeta_{2} \rightarrow 0$ there is at least one neighbourhood of zero which does not contain $\frac{\pi u_{0}[0]}{\beta} \zeta_{2}$. Both possibilities concerning $u_{0}[0]$ in the interior must be examined.

If $u_{0}[0]>>\frac{c}{\zeta_{2}}$ then at $\zeta_{2} \rightarrow 0$ the third and the sixth term in (68) also vanish identically. If, on the contrary, $u_{0}[0]$ is a positive constant then at $\zeta_{2} \rightarrow 0$ with $\zeta_{1} \neq 0$ the singular part $D_{a s}^{(i n t)}\left(x_{2}-x_{1}\right)$ of the scalar propagator diverges as $\zeta_{2}^{-\frac{1}{2}}$. Clearly, an appropriate boundary condition imposed by the physical context within which this situation occurs will determine which of these two eventualities is realised. In order to determine that boundary condition use must be made of the fact that - as stated in the beginning of this section - in the interior region of the Schwarzschild black hole the roles of space and time are reversed. This reversal can be directly elicited by the change which occurs in the signature of the Schwarzschild metric in (1) when passing from the region $r>2 M$ to the region $r<2 M$. In the interior region the Schwarzschild radial variable $r$ 
becomes time-like. As a result, the decrease of $r$ in the interior region labels the advance of time which is, for that matter, inexorably directed toward the time coordinate $r=0$.

As $r \rightarrow 2 M^{-}$the square of proper time's increment $d s^{2}$ in (1) tends to negative infinity. The imaginary character of the in-falling observer's proper time merely expresses the fact that his world line is unobservable to all observers in the region $r>2 M$. At once, the divergence in proper time admits the interpretation that, expressed in terms of the Schwarzschild time-like coordinate $r$, the time registered on the clock of an observer who has just crossed the event horizon advances at infinite rate. Consequently, in terms of the Schwarzschild time-like coordinate $r$, quantum propagation between two points in the interior region occurs instantaneously if one of the two points has been specified arbitrarily close to the event horizon $\left(r \rightarrow 2 M^{-}\right)$. As a consequence, described in terms of $r$ the corresponding transition amplitude must diverge.

The stated divergence in the transition amplitude reflects the corresponding divergence in the proper time of the in-falling observer who has just crossed the event horizon. Just like the latter so the divergence in the transition amplitude is unobservable in all circumstances. The unobservable divergence in the proper time itself is not merely a consequence of $\lim _{r \rightarrow 2 M^{-}}\left(1-\frac{2 M}{r}\right)=0$ but also of the fact that in the interior region the situation $d r=0$ is impossible. Consequently, although that divergence is specific to Schwarzschild coordinates it is, at once, a consequence of the coordinate-independent reversal which occurs in the interior region to the effect that what was space effectively becomes time and vice versa. As a result, the unobservable divergence which occurs in the transition amplitude away from the coincidence space-time limit is a consequence of the space-time geometry's causal structure on condition that quantum propagation is described in terms of Schwarzschild coordinates. Of course, from the perspective of the in-falling observer himself nothing unusual happens. Both, the lapse of proper time which his clock registers and the transition probability for quantum propagation which he (in principle) observes just after he has crossed the event horizon remain finite and well defined.

It can readily be seen, for that matter, that the boundary condition which the physical context imposes on (68) is that the only two terms which do not vanish at $\zeta_{2} \rightarrow 0$, namely the third and the sixth term, must diverge. Consequently, in these two terms $u_{0}[0]$ is a positive constant so that these two terms do, indeed, diverge as $\zeta_{2}^{-\frac{1}{2}}$. As a consequence of this as well as of (84) it follows that, at $\zeta_{2} \rightarrow 0$, the singular part $D_{a s}^{(i n t)}\left(x_{2}-x_{1}\right)$ diverges in its entirety as $\zeta_{2}^{-\frac{1}{2}}$ on condition that $\zeta_{1} \neq 0$. This, in turn, implies that

$$
\lim _{\zeta_{2} \rightarrow 0} D^{(i n t)}\left(x_{2}-x_{1}\right)=\infty ; \zeta_{1} \neq 0
$$

In passing, it is worth noting that the condition $u_{0}[0]>>\frac{c}{\rho_{2}}$ which is valid in the exterior region can also be established by invoking boundary conditions derived from the physical context. The boundary condition which, in this case, would preclude a $\rho_{2}$-independent $u_{0}[0]$ is that - as stated in (76) - from the perspective of the observer in the static region the scalar propagator must vanish if one end-point of propagation is specified arbitrarily close to the event horizon. However, since the mathematical context itself already precludes a $\rho_{2}$-independent $u_{0}[0]$ the mathematical resolution which $\mathrm{I}$ have 
presented is a superior approach in view of the fact that it eventuates in the physical interpretation of vanishing propagation in (76).

The asymptotic behaviour of the propagator expressed, in the interior and in the exterior region respectively, in (85) and (76) allows for the evaluation of the transition amplitude in (81). The latter is

$$
<\rho_{1}^{\prime}, \theta_{1}^{\prime}, \phi_{1}^{\prime}, \tau_{1}^{\prime} \mid i \zeta_{1}, i \theta_{1}, \phi_{1}, i \xi_{1}>=
$$

$$
\int_{-\pi}^{\pi} d \theta_{2} \int_{0}^{2 \pi} d \phi_{2}\left[D_{a s}\left(x_{1}^{\prime}-x_{2}\right)+D_{b}\left(x_{1}^{\prime}-x_{2}\right)\right]\left[D_{a s}^{(i n t)}\left(x_{2}-x_{1}\right)+D_{b}^{(i n t)}\left(x_{2}-x_{1}\right)\right]
$$

with $x_{1}=\left(i \xi_{1}, i \zeta_{1}, i \theta_{1}, \phi_{1}\right), x_{2}=\left(\rho_{2} \rightarrow 0, \theta_{2}, \phi_{2}\right)$ and $x_{1}^{\prime}=\left(\tau_{1}^{\prime}, \rho_{1}^{\prime}, \theta_{1}^{\prime}, \phi_{1}^{\prime}\right)$.

Terms which involve products between a singular part on either side of the event horizon and a boundary part on the other side do not contribute to the Green function on the left side of (86). The most expedient way to establish this in the present context in which integration occurs over the physically irrelevant angular sector is to note that whereas $D_{a s}^{(i n t)}\left(x_{2}-x_{1}\right)$ contains a singularity if the limit $x_{2} \rightarrow x_{1}$ is taken anywhere within the range stated in (74) the boundary part $D_{b}\left(x_{1}^{\prime}-x_{2}\right)$ remains finite if $x_{2} \rightarrow x_{1}^{\prime}$ is taken anywhere within the range stated in (8). Consequently, the term in (86) which involves the product $D_{a s}^{(i n t)}\left(x_{2}-x_{1}\right) D_{b}\left(x_{1}^{\prime}-x_{2}\right)$ contributes neither to the singular part of $<\rho_{1}^{\prime}, \theta_{1}^{\prime}, \phi_{1}^{\prime}, \tau_{1}^{\prime} \mid i \zeta_{1}, i \theta_{1}, \phi_{1}, i \xi_{1}>$ nor to the boundary part of that Green function. For the same reason the term in (86) which involves the product $D_{a s}\left(x_{1}^{\prime}-x_{2}\right) D_{b}^{(i n t)}\left(x_{2}-\right.$ $x_{1}$ ) has no contribution to $<\rho_{1}^{\prime}, \theta_{1}^{\prime}, \phi_{1}^{\prime}, \tau_{1}^{\prime} \mid i \zeta_{1}, i \theta_{1}, \phi_{1}, i \xi_{1}>$. Formally, these two "cross terms" in (86) cancel against the corresponding terms which are inherent in $D_{a s}\left(x_{1}^{\prime}-\right.$ $\left.x_{2}\right) D_{a s}^{(i n t)}\left(x_{2}-x_{1}\right)$. Specifically, $D_{a s}\left(x_{1}^{\prime}-x_{2}\right)$ and $D_{a s}^{(i n t)}\left(x_{2}-x_{1}\right)$ can always be expressed as $\tilde{D}_{a s}\left(x_{1}^{\prime}-x_{2}\right)+\tilde{D}_{f i n}\left(x_{1}^{\prime}-x_{2}\right)$ and $\tilde{D}_{a s}^{(i n t)}\left(x_{2}-x_{1}\right)+\tilde{D}_{f i n}^{(i n t)}\left(x_{2}-x_{1}\right)$ respectively in such a manner as to enforce $\left|\tilde{D}_{a s}\left(x_{1}^{\prime}-x_{2}\right) \tilde{D}_{f i n}^{(i n t)}\left(x_{2}-x_{1}\right)\right|=\left|D_{a s}\left(x_{1}^{\prime}-x_{2}\right) D_{b}^{(i n t)}\left(x_{2}-x_{1}\right)\right|$ and $\left|\tilde{D}_{a s}^{(i n t)}\left(x_{2}-x_{1}\right) \tilde{D}_{f i n}\left(x_{1}^{\prime}-x_{2}\right)\right|=\left|D_{a s}^{(i n t)}\left(x_{2}-x_{1}\right) D_{b}\left(x_{1}^{\prime}-x_{2}\right)\right|$ [4]. The boundary term $D_{b}\left(x_{1}^{\prime}-x_{2}\right)$ has the opposite sign to that of the finite term $\tilde{D}_{f i n}\left(x_{1}^{\prime}-x_{2}\right)$ in view of the fact that the former has been designed to enforce the boundary condition on the Green function in the exterior region. At once, the analytical extension of that Green function into the interior region implies that the boundary term $D_{b}^{(i n t)}\left(x_{2}-x_{1}\right)$ has the opposite sign to that of the finite term $\tilde{D}_{\text {fin }}^{(i n t)}\left(x_{2}-x_{1}\right)$.

Consequently, (86) reduces to

$$
<\rho_{1}^{\prime}, \theta_{1}^{\prime}, \phi_{1}^{\prime}, \tau_{1}^{\prime} \mid i \zeta_{1}, i \theta_{1}, \phi_{1}, i \xi_{1}>=
$$

$$
\int_{-\pi}^{\pi} d \theta_{2} \int_{0}^{2 \pi} d \phi_{2}\left[D_{a s}\left(x_{1}^{\prime}-x_{2}\right) D_{a s}^{(i n t)}\left(x_{2}-x_{1}\right)\right]+\int_{-\pi}^{\pi} d \theta_{2} \int_{0}^{2 \pi} d \phi_{2}\left[D_{b}\left(x_{1}^{\prime}-x_{2}\right) D_{b}^{(i n t)}\left(x_{2}-x_{1}\right)\right]
$$

A crucial aspect of this derivation is that the contribution of $D_{a s}^{(i n t)}\left(x_{2}-x_{1}\right)$ to the first term on the right side of (87) enters through the two non-singular terms which correspond 
to $|\tilde{p}| \rightarrow 0$ - that is, through the third and the sixth term in (68) - since it is only these two terms which do not vanish at $\zeta_{2} \rightarrow 0$. Of course, at the coincidence space-time limit $x_{2} \rightarrow x_{1}$ in the interior and on the event horizon it is the remaining four terms in (68) which are responsible for the quadratic divergence of $<\rho_{1}^{\prime}, \theta_{1}^{\prime}, \phi_{1}^{\prime}, \tau_{1}^{\prime} \mid i \zeta_{1}, i \theta_{1}, \phi_{1}, i \xi_{1}>$.

In the interior vicinity of the event horizon $\left(\zeta_{2}<<1\right)$ the cause of the divergence at $\zeta_{2} \rightarrow 0$ in (85) is, as stated, the factor $\zeta_{2}^{-\frac{1}{2}}$ in the third and the sixth term of (68). In the exterior vicinity of the event horizon $\left(\rho_{2}<<1\right)$ the singular part $D_{a s}\left(x_{1}^{\prime}-x_{2}\right)$ in $(6)$ behaves as [4]

$$
\begin{gathered}
\lim _{\rho_{2} \rightarrow 0} D_{a s}\left(x_{1}^{\prime}-x_{2}\right)=C \frac{1}{2 \pi^{2}} \frac{1}{\beta^{2}} \times \\
\lim _{\rho_{2} \rightarrow 0} \frac{\sqrt{\rho_{2}}}{\sqrt{\rho_{1}}} \sum_{k=0}^{\infty} \sum_{p \neq 0}^{\infty} e^{i \frac{p}{\beta}\left(\tau_{2}-\tau_{1}\right)} \frac{2 k+1}{p} P_{k}(\cos \gamma) ; \rho_{1}^{\prime} \neq 0 ; C<<1 ; \tau_{2} \rightarrow \infty
\end{gathered}
$$

Consequently, in the integrand $D_{a s}\left(x_{1}^{\prime}-x_{2}\right) D_{a s}^{(i n t)}\left(x_{2}-x_{1}\right)$ of the first term in (87), the factor $\zeta_{2}^{-\frac{1}{2}}$ cancels against the factor $\rho_{2}^{\frac{1}{2}}$ prior to $\left|\rho_{2}\right| \rightarrow 0$. As a result, the transition amplitude $<\rho_{1}^{\prime}, \theta_{1}^{\prime}, \phi_{1}^{\prime}, \tau_{1}^{\prime} \mid i \zeta_{1}, i \theta_{1}, \phi_{1}, i \xi_{1}>$ is a regular and non-vanishing function of space-time. The "uncertainty principle" has canceled the vanishing effect expressed in (76). Within a finite advance of his proper time $t$ the observer situated at point $A$ in Fig.6 registers radiation emitted by the Schwarzschild black hole - as promised!

\section{Conclusions and Discussion}

Through an appropriate analytical extension performed on the massless conformal scalar propagator obtained in [4] the entire analysis herein eventuated in an analytic expression for that propagator in the interior region of the Schwarzschild black-hole geometry. For a specific range of values of the Schwarzschild radial coordinate that expression is a valid approximation to the exact massless conformal scalar propagator associated with the Hartle-Hawking vacuum state in the interior region. Being an analytic expression the stated propagator is explicit in its dependence on space-time in the interior region of the Schwarzschild black hole. This approximation to the exact propagator is, therefore, unique.

As is also the case with the results in [4] an essential advantage of the propagator which has been obtained herein is that its short-distance behaviour and singularity structure are manifest. Despite its complicated structure this propagator is, for that matter, especially suited for an analytic evaluation of $\left\langle T_{\mu \nu}\right\rangle$ and $\left\langle\phi^{2}(x)>\right.$ as well as for perturbative calculations in the interior of the black hole. In [4] the renormalised value of $\left\langle\phi^{2}(x)>\right.$ was obtained in the Hartle-Hawking vacuum state in the exterior region of the Schwarzschild black hole. It would be desirable to demonstrate the capacity of the propagator obtained herein for actual calculations in perturbative renormalisation by extending that evaluation to the evaluation of the renormalised value of $\left\langle\phi^{2}(x)>\right.$ in the interior region of the black hole and compare it against the corresponding results in [2]. Such a task is contingent upon the consistent formulation of a computer program which will produce the relevant graphs. 
In [4] the range of values stated in (8) was shown to signify a range of validity for the approximation which corresponds to several orders of magnitude above the range within which particles are spontaneously created and back-reaction effects are pronounced. The relation of the range expressed in (74) to the physical content in the interior of the black hole as well as the effect which the causal structure of the interior space-time geometry has to scalar propagation have been explored in section VI. The range of validity expressed in (74) is of the same order of magnitude as that expressed in (8). Just as, for that matter, the latter signifies an ample range of values of $r>2 M$ within which the approximation to the exact propagator is valid [4] it is reasonable to state that (74) also signifies an ample range of validity in the interior.

An issue of central importance is the match of the propagator obtained herein in the interior region with that obtained in [4 in the exterior region, across the event horizon. That task was accomplished in section VI. It was shown that, as a result of that match, the massless scalar propagator which corresponds to the Hartle-Hawking vacuum state results in particle production by the Schwarzschild black hole which is registered by static observers situated within the range of the propagator's validity and - by extension - by any static observer at any distance from the event horizon. In order to show that the particle production which this propagator describes coincides with Hawking radiation it is also necessary to show that that particle production has a thermal spectrum which corresponds to the relevant surface-gravity related temperature. That, in turn, may be established by showing that the probability for the emission of a massless scalar particle of a certain energy and the absorption of a massless scalar particle of the same energy relate to each other in the manner established in [9]. Past that point the task will be the exploration of the relation which higher-order radiative effects in the presence of self-interactions of the $\phi^{4}(x)$-type have to the thermal character of the black-hole radiation in the Hartle-Hawking vacuum state. Being especially suited for perturbative calculations the massless scalar propagator obtained in this project has the potential to advance such an exploration although, at once, in view of the highly complicated expression which the massless conformal scalar propagator receives in the interior region of the Schwarzschild black hole this may impress as a formidable task. Nevertheless, the derivation of particle production in section VI is indicative of the fact that a careful approach to the causal structure of the Schwarzschild black-hole space-time can substantially simplify the calculations.

It is my intension to address these issues.

\section{Acknowledgements}

I wish to thank my wife Minnie for her unsolicited patience.

\section{REFERENCES}

[1] P. Candelas, Phys. Rev. D21, 2185, (1980).

[2] P. Candelas and B. P. Jensen, Phys. Rev. D33, 1596, (1986)

[3] U. A. Yajnik and K. Narayan, Class.Quant.Grav. 15, 1315-1321, (1998) ; e-Print: gr-qc/9706021.

[4] G. Tsoupros, Gen.Rel.Grav. 42, 1103-1138, (2010) ; e-Print: arXiv:0909.4886 [hep-th].

[5] S. W. Hawking, Commun. Math. Phys. 80, 421-442, (1981)

[6] F. W. J. Olver, Asymptotics And Special Functions, Academic Press, (1974) 
[7] P. Candelas and B. P. Jensen, Phys. Rev. D33, 1590, (1986)

[8] R. Courant and D. Hilbert, Methods Of Mathematical Physics, John Wiley and Sons, (1989) - (p. 512, vol. I)

[9] J. B. Hartle and S. W. Hawking, Phys. Rev. D13, 2188, (1975)

[10] N. D. Birrell and P. C. W. Davies, Quantum Fields in Curved Space, Cambridge Monographs in Mathematical Physics, (1984), p. 280. 